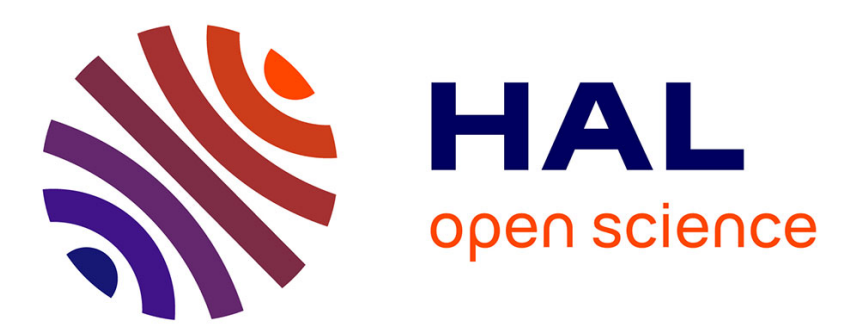

\title{
Une marchandise scientifique? Savoirs, industrie et régulation du médicament dans l'Allemagne des années trente
}

Jean-Paul Gaudillière

\section{- To cite this version:}

Jean-Paul Gaudillière. Une marchandise scientifique? Savoirs, industrie et régulation du médicament dans l'Allemagne des années trente. Annales. Histoire, Sciences sociales, 2010, 65 (1), pp.89-120. halshs-00683128

\section{HAL Id: halshs-00683128 \\ https://shs.hal.science/halshs-00683128}

Submitted on 19 Jan 2022

HAL is a multi-disciplinary open access archive for the deposit and dissemination of scientific research documents, whether they are published or not. The documents may come from teaching and research institutions in France or abroad, or from public or private research centers.
L'archive ouverte pluridisciplinaire HAL, est destinée au dépôt et à la diffusion de documents scientifiques de niveau recherche, publiés ou non, émanant des établissements d'enseignement et de recherche français ou étrangers, des laboratoires publics ou privés. 


\title{
Une marchandise scientifique? Savoirs, industrie et régulation du médicament dans l'Allemagne des années trente
}

\author{
Jean-Paul Gaudillière
}

Produire en masse des agents thérapeutiques, les breveter, les empaqueter pour les acheminer vers les pharmacies, en négocier le prix avec l'assurance-maladie, faire leur publicité auprès des médecins, les rappeler en cas d'effets indésirables dus à un vice de forme ou pire à des propriétés imprévues des substances actives. Tout cela appartient à l'ordinaire actuel de la construction des marchés pharmaceutiques. Au début du XXe siècle, l'ordinaire de la marchandise "médicament " était pourtant très différent. Le pharmacien n'est pas un épicier, répétaient à l'envie aussi bien les professeurs de faculté que les associations de la pharmacie et de la droguerie.

Plus, dans nombre de pays, les titulaires de diplôme s'opposaient - au nom de la compétence, de la science et de l'expertise - à toute libéralisation qui faciliterait la mise sur le marché tant des spécialités dites scientifiques ou éthiques - une façon pour leurs producteurs de signaler qu'ils en garantissaient et rendaient publique la composition - que des « remèdes secrets ». Entre le tournant du siècle et la Première Guerre mondiale, l'Union des apothicaires allemands tenta ainsi, au nom de la responsabilité du pharmacien en matière de sécurité des médicaments, et sans grand succès, d'imposer une obligation de fabrication à l'officine qui aurait - de fait - rendue illégale la vente de l'ensemble des spécialités industrielles dites galéniques - comprimés compris ${ }^{1}$. 
L'histoire du médicament et de la pharmacie n'est donc pas un domaine vierge de l'histoire du XX $x^{\mathrm{e}}$ siècle. Loin s'en faut. Cette historiographie a toutefois longtemps privilégié deux types d'approches, poursuivies en parallèle. La première était celle des grandes découvertes de la pharmacie comme science; une science à forte dose de chimie, une science de laboratoire dont les hauts faits ont d'abord été ceux de l'analyse et de l'isolement des substances contenues dans les «matières médicales » avant de se consacrer à la synthèse de nouvelles molécules, aux essais de leurs propriétés non plus seulement chez l'animal mais aussi chez l'homme. La seconde était celle de la profession et de l'économie du médicament, une histoire de corporation et de segments professionnels profondément remodelés par l'industrialisation et la production de masse, par la concentration et l'émergence d'un capitalisme pharmaceutique opérant sur un marché très particulier, fortement encadré par l'État et les assurances, alternativement pris comme modèle soit d'une économie de l'innovation, soit au contraire de la construction de monopoles. Entre les deux, le lien était plus souvent postulé qu'exploré, généralement associé à l'idée de «révolution thérapeutique», un changement radical de la nature et des usages des agents thérapeutiques qui intervint durant les «trente glorieuses» banalisa le médicament-marchandise-produit de la recherche industrielle.

L'historiographie de la seconde moitié du siècle est ainsi paradoxalement nettement plus développée que celle portant sur la période 1870-1945. Elle a certainement bénéficié du statut scientifique et médical des nouvelles chimiothérapies, mais sans doute aussi de l'interface avec les sciences sociales, qu'il s'agisse de la sociologie de la santé, des professions ou plus récemment de l'anthropologie et des études sociales des sciences.

Sans tenter une présentation d'ensemble, il faut insister sur deux points forts de la littérature récente. Le premier est l'exploration de l'histoire d'entités thérapeutiques dont les «biographies» permettent non seulement d'explorer des pratiques d'invention très hétérogènes mais aussi les interactions entre les mondes de la science, de la médecine, du droit, de la politique. Au nombre des innovations ayant bénéficié d'un traitement important, il faut ainsi compter non seulement la pénicilline ou la streptomycine, mais aussi les sulfamides, les hormones sexuelles et la pilule, le vaccin polio, certains anticancéreux, les corticoïdes, les tranquillisants, antidépresseurs et autres psychotropes ${ }^{2}$. Un second point fort est l'exploration des interventions de l'État, des changements du statut légal des médicaments et de la législation pharmaceutique. La période d'après 1945 est en effet celle durant

2 - Robert Bud, Penicillin, triumph and tragedy, Oxford, Oxford University Press, 2007; John E. LEsch, The first miracle drug: How the sulfa drugs transformed medicine, New York, Oxford University Press, 2007; Viviane Quirke, Collaboration in the pharmaceutical industry: Changing relationships in Britain and France, 1935-1965, Londres, Routledge, 2008; Nelly Oudshoorn, Beyond the natural body: An archeology of sex hormones, Londres, Routledge, 1994; David Healy, Le temps des antidépresseurs, Paris, Les Empêcheurs de penser en rond, [1997] 2002; Jordan Goodman et Vivien WaLSh, The story of taxol: Nature and politics in the pursuit of an anti-cancer drug, Cambridge, Cambridge University Press, 2001. 
laquelle la délégation d'expertise globalement accordée aux professions de santé pour évaluer la qualité, la sûreté et l'utilité des agents thérapeutiques a été profondément modulée par la multiplication des dispositifs de régulation administrative : agences, permis de mise sur le marché ou visa, tests standards, protocoles d'essais, recommandations d'usage, etc. Les réformes de la Food and Drug Administration (FDA) américaine ont tout particulièrement attiré l'attention, ne serait-ce que parce que le gouvernement américain a été le premier, en 1962, à transformer les essais cliniques dits contrôlés, c'est-à-dire organisés selon les règles préconisées par les statisticiens, en outil légal ${ }^{3}$. Les rapports entre pharmaciens, firmes et État, la régulation prise comme phénomène institutionnel, occupent donc une part importante de la littérature. Les travaux sur les pratiques industrielles sont eux - pour partie pour des raisons d'accès aux sources - beaucoup moins nombreux. Il en est ainsi de l'histoire de la recherche industrielle qui a surtout été abordée du point de vue d'une histoire économique interrogeant la réussite ou l'échec dans la mise au point de composés. De même, les rapports entre firmes et médecins, la place du travail thérapeutique sont des enjeux souvent mentionnés mais rarement analysés ${ }^{4}$.

L'objectif de cet article est de revenir sur la double identité du médicament comme marchandise et comme objet de science en partant d'une configuration spécifique, celle de l'entre-deux-guerres, moment où l'industrialisation de la pharmacie, les savoirs mobilisés pour préparer les médicaments, la façon de juger de leurs effets, les relations entre hommes de laboratoire, industriels, pharmaciens et médecins sont en pleine recomposition, font l'objet de controverses ou de conflits ouverts. Compte tenu de ce qui vient d'être dit sur l'historiographie des quinze dernières années, prétendre lier histoire des sciences, histoire des techniques et histoire de la médecine ne saurait suffire. La spécificité du point de vue proposé ici tient plutôt au fait d'aborder les activités des firmes, des chercheurs et des médecins par les divers registres de savoirs qu'ils ou elles mobilisent, autrement dit d'essayer de tenir ensemble des activités d'expérimentation, de production, de marketing et de soin. On les abordera en analysant de façon détaillée les pratiques associées à deux «formes de régulation» du médicament.

3 - Arthur A. Daemmerich, Pharmacopolitics: Drug régulation in the United States and Germany, Chapel Hill, University of North Carolina Press, 2004; Harry M. MARKs, La médecine des preuves. Histoire et antropologie des essais cliniques, 1900-1990, Paris, Les Empêcheurs de penser en rond, [1997] 1999; John P. Swann, Academic scientists and the pharmaceutical industry: Cooperative research in twentieth century America, Baltimore, Johns Hopkins University Press, 1988. Pour la France, voir Sophie Chauveau, L'innovation pharmaceutique. La pharmacie française entre l'État et la société au XX $X^{e}$ siècle, Paris, Les Empêcheurs de penser en rond, 1999; Christian BONAH et Anne Rasmussen (dir.), Histoire et médicament aux XIX et XX $X^{e}$ siècles, Paris, Biotem/Éd. Glyphe, 2005.

4 - Parmi les exceptions, Christiane SINDING, «Making the unit of insulin: Standards, clinical work, and industry, 1920-1925 ", Bulletin of the History of Medicine, 76, 2002, p. 231-270; Nicolas RASMUSSEN, «The moral economy of the drug company - medical scientists collaboration in interwar America ", Social Studies of Science, 34-2, 2004, p. 161-185 et Jeremy A. Greene, Prescribing by numbers: Drugs and the definition of diseases, Baltimore, Johns Hopkins University Press, 2007. 


\section{La régulation du médicament}

Le terme de régulation est le plus souvent utilisé dans un sens légal et administratif, pour désigner les dispositifs étatiques de contrôle du marché et de la qualité des produits. On adoptera ici une définition plus large de la régulation comme un ensemble de pratiques réglant la caractérisation, la production, la circulation, les usages des médicaments; des pratiques qui peuvent aussi bien être le fait de l'État, des fabricants, des professionnels de santé que des patients; des pratiques non réductibles aux seuls corpus de règles formelles mais qui font intervenir des choix de valeurs et d'enjeux prioritaires, des formes d'administration de la preuve, des lieux et des outils spécifiques d'intervention. Dans cette perspective, les trajectoires des médicaments au cours du $\mathrm{XIX}^{\mathrm{e}}$ et $\mathrm{du} \mathrm{XX}^{\mathrm{e}}$ siècle s'inscrivent dans une histoire de l'apparition et du changement des hiérarchies entre formes de régulation (voir tableau). Plus spécifiquement, la crise de la matière médicale évoquée au début de cet article peut être lue comme une manifestation de l'érosion de la régulation professionnelle dominante avant 1900 au profit d'une régulation industrielle.

Quatre formes de régulation du médicament au $x x^{\mathrm{e}}$ siècle

\begin{tabular}{|c|c|c|c|c|}
\hline $\begin{array}{c}\text { Forme } \\
\text { de régulation }\end{array}$ & Professionnelle & Industrielle & Administrative & $\begin{array}{l}\text { Consumériste- } \\
\text { civique }\end{array}$ \\
\hline $\begin{array}{l}\text { Valeurs, } \\
\text { objectifs mis } \\
\text { en avant }\end{array}$ & $\begin{array}{l}\text { Compétence, } \\
\text { compliance }\end{array}$ & $\begin{array}{l}\text { Productivité, profit, } \\
\text { qualité }\end{array}$ & $\begin{array}{l}\text { Santé publique, } \\
\text { efficacité clinique, } \\
\text { accès }\end{array}$ & $\begin{array}{l}\text { Choix individuel, } \\
\text { qualité de vie, } \\
\text { contrôle } \\
\text { des risques }\end{array}$ \\
\hline $\begin{array}{l}\text { Formes } \\
\text { de preuve } \\
\text { acceptées }\end{array}$ & $\begin{array}{l}\text { Pharmacologie, } \\
\text { courbe dose-effet, } \\
\text { classification } \\
\text { des maladies }\end{array}$ & $\begin{array}{l}\text { Essais standards } \\
\text { (chimiques et } \\
\text { biologiques), } \\
\text { études de marché }\end{array}$ & $\begin{array}{l}\text { Essais cliniques } \\
\text { contrôlés, } \\
\text { statistiques }\end{array}$ & $\begin{array}{l}\text { Épidémiologie, } \\
\text { observations } \\
\text { post-marketing, } \\
\text { analyses } \\
\text { risque-bénéfices }\end{array}$ \\
\hline $\begin{array}{l}\text { Arènes } \\
\text { de référence }\end{array}$ & $\begin{array}{l}\text { Sociétés savantes, } \\
\text { associations } \\
\text { corporatives }\end{array}$ & $\begin{array}{l}\text { Firmes, } \\
\text { associations } \\
\text { de producteurs, } \\
\text { syndicats }\end{array}$ & $\begin{array}{l}\text { Agences étatiques, } \\
\text { comité d'experts }\end{array}$ & $\begin{array}{l}\text { Associations } \\
\text { de patients et de } \\
\text { consommateurs, } \\
\text { ONG, médias }\end{array}$ \\
\hline $\begin{array}{l}\text { Instruments } \\
\text { de régulation }\end{array}$ & $\begin{array}{l}\text { Pharmacopées, } \\
\text { prescription, } \\
\text { recommandations } \\
\text { de pratiques }\end{array}$ & $\begin{array}{l}\text { Contrôle de } \\
\text { qualité, marketing } \\
\text { scientifique }\end{array}$ & $\begin{array}{l}\text { Permis de } \\
\text { commercialisation, } \\
\text { notices, } \\
\text { liste d'indications }\end{array}$ & $\begin{array}{l}\text { Campagnes } \\
\text { d'information, } \\
\text { lobbying politique, } \\
\text { décisions } \\
\text { de justice }\end{array}$ \\
\hline
\end{tabular}

La régulation professionnelle a dominé le monde de la pharmacie pendant tout le XIXe siècle, mais elle ne disparaît pas avec celui-ci. Elle trouve ses racines dans l'organisation professionnelle de la formation et de l'exercice telle que l'a définie la loi française de 1808 ou la loi allemande de 1871 mais surtout, du point de vue des savoirs, par une délégation d'expertise laissant aux instances professionnelles le soin de prendre les décisions regardant l'élaboration, la qualification et l'accès aux produits. Cette régulation professionnelle n'était donc pas seulement 
un moyen de réguler l'offre en évitant l'entrée sur le marché de compétiteurs non qualifiés et en nombre, mais également un dispositif de contrôle des agents thérapeutiques dont le point de départ était la culture de la préparation et la principale cible la question de la toxicité. La responsabilité professionnelle - individuelle comme collective - consistait en effet à garantir la préparation et, avec elle, l'absence d'intoxication dans les conditions normales d'usage. Dans cette perspective, le savoir de référence est pharmacologique: il porte sur les relations entre dosage administré et action physiologique, sur la concentration des produits dans le corps et les voies de leur élimination, sur la frontière entre effets thérapeutiques et effets toxiques. Les outils privilégiés sont les modèles animaux (de la maladie ou de la physiologie normale) et les courbes dites doses-réponses. La routine de la régulation consiste alors pour les instances professionnelles pharmaceutiques et médicales à définir des pratiques de référence comme les protocoles d'identification ou de dosage, comme les listes d'indications ou de contre-indications, le statut de ces listes pouvant varier entre l'obligation légale et la simple recommandation corporative.

Une remarquable illustration de la combinaison de technologies matérielles, sociales et littéraires caractéristique de cette régulation professionnelle est fournie par l'histoire des pharmacopées. Bien qu'elles aient existé auparavant, celles-ci changent de statut au XIX ${ }^{\mathrm{e}}$ siècle pour devenir des instruments légaux dont l'existence est indissociable de la montée des pouvoirs de l'État-nation en matière d'expertise technique et de régulation des marchés ${ }^{5}$. Les pharmacopées ont eu des frontières variables - tant en ce qui concerne les préparations que les types de savoir-faire intégrés - mais toutes celles qui ont acquis le caractère de pharmacopée «nationale» ont eu pour fonction de dresser une liste des médicaments officiellement reconnus : les agents thérapeutiques inclus dans les codex ont rang de médicament, peuvent être prescrits par les médecins et vendus par les pharmaciens. L'ambiguïté de cette reconnaissance tenait toutefois à la difficulté à exclure certains produits de la vente puisque la liste n'était pas exclusive, les autres préparations n'étant pas interdites. Ceci n'impliquait évidemment pas que l'inclusion dans la pharmacopée n'avait pas de conséquences, mais seulement que celle-ci constituait non pas le précurseur d'une autorisation de mise sur le marché mais une forme de reconnaissance de la valeur médicale d'une préparation dont la sanction était la diffusion de la recette par le biais de l'enseignement et de l'édition.

En pratique, la principale fonction des pharmacopées était toutefois d'organiser la pratique d'officine en constituant des livres des recettes de référence utilisées soit pour la préparation des substances thérapeutiques, des extraits et mélanges, soit pour le contrôle tant des matières premières indispensables à la fabrication des extraits et mélanges que des produits fournis par l'officine. Sans rupture avec les fonctions qu'elles avaient dans la culture corporative, les pharmacopées nationales du XIX ${ }^{\mathrm{e}}$ siècle sont en premier lieu des index de préparations qui récapitulent origine, 
propriétés caractéristiques, modalités de production d'une «matière médicale » dans laquelle les plantes jouent un rôle bien plus important que les substances chimiques purifiées. Il s'agissait d'un compendium de protocoles de travail qui furent les outils privilégiés d'une standardisation professionnelle combinant des dimensions de classification (dire la différence entre préparations), d'identification matérielle (donner les caractéristiques d'une substance et les tests de référence pour l'identifier) et d'organisation du travail à l'officine (donner les étapes et le détail des opérations nécessaires pour produire une formulation thérapeutique). Cette dimension pratique de la standardisation par et pour la profession était indissociable de la régulation du marché et de la gestion quotidienne du monopole d'exercice. Ceci est évident dans les pays où, comme en Allemagne, la loi faisait obligation au pharmacien - d'officine ou d'entreprise - de respecter les recettes du livre des médicaments quand elles existaient. Mais même là où, comme en France, la réglementation était plus ambiguë et laissait une certaine marge de manœuvre au pharmacien préparateur, l'écriture du mode de préparation officiel restait un des moyens de décider qui pouvait préparer et commercialiser quoi.

L'hypothèse défendue ici est qu'au tournant du XX⿳亠丷厂 siècle, un autre type de régulation a pris forme, centré cette fois sur la pratique industrielle. La régulation industrielle n'a pas éliminé la régulation professionnelle. Elle a opéré pour partie en parallèle, pour partie en remplacement de cette dernière. Son émergence a modifié les interventions de la profession et de l'État. Le terreau de cette régulation industrielle est la transformation des officines-ateliers en usines, le passage à la production de masse de spécialités et pas seulement l'entrée sur le marché des agents thérapeutiques de grandes firmes de la chimie industrielle. Ce changement de la nature des produits est à l'origine de tensions très importantes touchant à la légitimité et la qualité des spécialités. Pour les atténuer si ce n'est les résoudre, la réponse des entreprises industrielles a été d'organiser et de mettre en avant la standardisation et le contrôle des compositions. Les dispositifs correspondants avaient en effet l'intérêt de lier deux objectifs : maximiser la création de valeur économique en contribuant aux augmentations de productivité, et éviter les effets négatifs sur la réputation des firmes des plaintes de pharmaciens et de médecins, des retours de préparations, voire des demandes de réparation et actions en justice. La mise en œuvre de cette régulation industrielle est passée par la création de départements ou de services internes de développement et surveillance associant des fonctions d'investigation pour élaborer des protocoles standards de production et de test, ainsi que par la mise en œuvre des dispositifs de contrôle des matières premières ou des produits finis. Les formes de savoir mobilisées ne se limitent donc pas à la chimie et aux manipulations des molécules, les essais physiologiques - de masse et standardisés - sont des éléments essentiels de ces dispositifs. Cette évolution, particulièrement marquée dans l'entre-deux-guerres, est inséparable du mouvement de «rationalisation» associé au nom de Frederick Taylor. Une dimension propre au secteur de la pharmacie concerne toutefois les modalités de construction des marchés. Les grandes firmes productrices de spécialités ont en effet progressivement recentré les cibles de ce qui s'appelle encore "propagande ", des patients et pharmaciens vers les médecins, liant les activités publicitaires avec 
la production et la circulation des informations scientifiques sur les propriétés des spécialités et de leurs usages. Autrement dit, la régulation industrielle passe aussi par une invention du «marketing scientifique » dont la publication de journaux de compagnies imitant les publications académiques ou la généralisation du système des visiteurs médicaux sont de bons marqueurs.

Explorer l'histoire du médicament au $\mathrm{XX}^{\mathrm{e}}$ siècle suppose bien évidemment de ne pas en rester au jeu des régulations professionnelles et industrielles, mais de prendre en compte la montée en puissance, durant la seconde moitié du siècle, de deux autres formes de régulation, administrative d'une part et consuméristecivique d'autre part (voir tableau). Leur analyse sort du cadre de cet article. On signalera seulement que la première est liée aux formes d'intervention de l'État classiquement associées à la régulation du médicament, en premier lieu les permis de mise sur le marché et l'évaluation de l'efficacité des produits sur la base d'essais cliniques contrôlés réalisés par les industriels. La seconde participe des transformations récentes de l'expertise avec les nouvelles formes d'action des associations d'usagers ou de patients et la multiplication des controverses publiques ou des actions judiciaires concernant les risques et les effets «indésirables» induits par la consommation massive de certains médicaments.

Concrètement, pour aborder ces enjeux, le texte qui suit part de l'histoire de deux firmes allemandes, Madaus et Schering, dont le positionnement, les stratégies, les marchés étaient radicalement différents et explore les trajectoires de deux types de médicaments issus du vivant: les extraits de plantes et les hormones sexuelles. Ce choix tient au fait que l'Allemagne est aujourd'hui un des rares «terrains » où l'accès aux archives de la pharmacie industrielle - ou du moins à certaines d'entre elles - est possible, si ce n'est facile. Mais il s'explique aussi par le caractère particulièrement visible dans ce pays des tensions entre culture de la préparation et production de spécialités industrielles. Comme chacun sait, l'Allemagne est au début du $\mathrm{Xx}^{\mathrm{e}}$ siècle la patrie de la grande industrie chimique et pharmaceutique, l'horizon vers lequel jeunes chimistes, biochimistes ou pharmacologues se tournent pour aller compléter leur formation. Mais c'est aussi le pays où, depuis les débuts de la République de Weimar, la médecine académique est considérée comme en crise, où ses paradigmes (en particulier la causalité bactérienne) sont tenus pour fragiles, où la mise en abyme des savoirs trouve ses racines dans un mouvement aussi diversifié que puissant de pratiques alternatives relevant d'une médecine «biologique» qui hérite de la culture de la matière médicale et parvient, dans les années 1930, à des formes de reconnaissance institutionnelle.

\section{Madaus et l'industrialisation des préparations de plantes}

L'exemple de la firme Madaus combine deux registres de discours et de pratiques que l'on a l'habitude de tenir pour incompatibles. Madaus était en effet, dans l'Allemagne des années 1920 et 1930, la principale firme productrice d'extraits et de mélanges à base de plantes médicinales, mais aussi un des centres les plus actifs dans la défense des thérapies biologiques et alternatives en opposition au 
réductionnisme de la médecine dite de faculté (Schulmedizin). L'historien de la médecine Carsten Timmermann a retracé l'histoire de la firme fondée par les trois frères Madaus, en insistant sur les relations d'empathie mais aussi de conflit que ses responsables ont entretenu avec le puissant mouvement de critique de la médecine allopathique qui caractérise la scène médicale allemande entre le début du siècle et la Seconde Guerre mondiale ${ }^{6}$.

Madaus a en effet été créée en tant qu'entreprise commerciale en 1919 pour vendre des remèdes homéopathiques et végétaux. Dans les années 1920, ces ventes connaissent une croissance très importante. Madaus ouvre des filiales dans de nombreuses villes tant allemandes qu'européennes, embauche massivement (elle compte 800 employés à la veille de la guerre) et s'engage dans une industrialisation de la fabrication qui a changé non seulement l'échelle mais aussi la nature des préparations. Le ressort de cette croissance n'est pas pour autant une culture de l'innovation pharmaceutique au sens de l'invention permanente de nouveaux produits. Il s'agit plutôt de revisiter, modifier et améliorer un ensemble de compositions et de recettes souvent connues, utilisées de façon traditionnelle par les praticiens locaux et négligées par les pharmaciens et médecins académiques. Sous l'impulsion de Gerhard Madaus, la firme développe ainsi un ensemble de pratiques de «modernisation» des préparations qui se distinguent aussi radicalement de celles des thérapeutes (Heilpraktiker) et médecins alternatifs que des formes d'innovation des firmes misant sur l'industrialisation par la chimie et la purification.

Dans les nombreux documents destinés à promouvoir la firme, ses produits et son corpus de connaissances, l'industrialisation et la scientificisation des préparations sont ainsi associées à une double argumentation. La première met en avant tout à la fois les symptômes plutôt que les entités nosographiques, la dimension constitutionnelle des maladies qui ne peuvent se comprendre et être traitées qu'à l'échelle de l'ensemble de l'organisme, l'importance des traitements biologiques respectueux des activités physiologiques, efficaces à faibles doses contrairement aux traitements chimiques, ou encore - nous y reviendrons - les bénéfices d'un recours aux plantes entières plutôt qu'aux substances actives isolées:

La reconnaissance par Virchow de la localisation (cellulaire) des pathologies est encore aujourd'hui d'une signification essentielle même si cette localisation est du point de vue biologique désormais tenue pour symptôme... Mais il est en fin de compte indifférent de savoir si une pathologie est en apparence limitée à un nombre restreint d'organes ou de systèmes. Le diagnostic spécifique indiquant que telle ou telle glande sécrétrice, le système immunitaire, le système circulatoire et lymphatique ou le système réticulo-endothélial sont touchés ne révèle en effet rien de la façon dont l'ensemble de l'organisme est en fait impliqué.

6 - Carsten Timmermann, «Rationalizing 'Folk medicine' in interwar Germany: Faith, business and science at 'Dr Madaus \& Co'”, Social History of Medicine, 14-3, 2001, p. 459-482; Robert JÜTTE, Geschichte der alternativen Medizin. Von der Volskmedizin zu den unkonventionellen Therapien von heute, Munich, Beck, 1996; Karl E. RoTschuH, Naturheilbewegungen, Reformbewegung, Alternativbewegung, Stuttgart, Hippokrates-Verlag, 1983. 
La question des interactions entre systèmes, de leurs relations aux développements de pathologie au niveau de l'organique isolé est au cour de la recherche moderne sur les constitutions et est encore loin d'être clarifiée. Il en est de même de l'influence des thérapies ${ }^{7}$.

Ces perspectives biologiques n'avaient toutefois pas pour conséquence de remettre en cause la production à grande échelle de préparations homogènes et prêtes à l'emploi. Le biologique tel que défini par G. Madaus est en effet une notion frontière qui permet de tenir ensemble et de donner une signification locale à des éléments issus tout à la fois du corpus classique de matière médicale, d'une nouvelle physiologie qui privilégie des effecteurs moléculaires comme les hormones et les vitamines, et d'une médecine populaire par les plantes. Le second registre est celui de l'industrialisation. Madaus est, jusqu'à la Seconde Guerre mondiale, une entreprise pharmaceutique florissante et les mérites que ses dirigeants attribuent à l'industrialisation ne se démarquent pas de ceux d'autres firmes sans lien avec la médecine alternative, comme Boehringer ou Gehe, elles aussi engagées dans la mobilisation de la pharmacopée pour une production de masse. L'enjeu de l'industrialisation est de produire plus pour moins cher (de façon à satisfaire une demande jugée croissante) tout en garantissant une pratique médicale plus efficace grâce à l'homogénéité de composition des produits, à leur stabilité et leurs capacités de conservation, à leur facilité de stockage et de transport. Homogénéité, standardisation et contrôle de qualité sont les trois termes d'un dispositif apparemment contradictoire avec les notions de prise en compte des constitutions individuelles des patients, de variabilité du cours suivi par la maladie et donc d'adaptation des formulations ou des prescriptions à chaque individu; notions toutes tenues pour essentielles par la médecine critique de l'entre-deux-guerres, et en particulier par la littérature homéopathique. De fait, le catalogue de Madaus comprend certes un grand nombre de préparations de plantes isolées - digitale, valériane, menthe, etc. pouvant être associées dans une prescription, mais aussi des complexes destinés au traitement d'une maladie ou d'un groupe de symptômes, comme cet Arnica Oligoplex traitant des infections avec forte fièvre, grippe incluse, et contenant un mélange d'extraits d'arnica, de belladone, de china, d'eucalyptus, et de noix vomique ${ }^{8}$.

Ces deux registres se rejoignent toutefois dans la critique de la pureté et la mise en avant des préparations complexes. Non seulement Madaus situait ses produits dans la continuité de l'art de la formulation, mais la firme a progressivement élaboré un discours sur la scientifisation des préparations qui était centré sur la complexité biologique et l'importance des mélanges. Ce discours mettait en avant plusieurs arguments: la composition globale faisant intervenir des dizaines de substances des extraits de plantes; l'impossible réduction de leurs effets à un

7 - Gerhard Madaus, Lehrbuch der biologischen Heilmittel, Leipzig, Georg Thieme Verlag, 1938, vol. I, p. 12. Il s'agit d'un des nombreux volumes ou brochures scientifiques publiés dans l'entre-deux-guerres à l'initiative de G. Madaus ou de la firme.

8 - Gerhard Madaus, Taschenbuch für die biologische Praxis, Dresde, Dr Madaus \& Co, 1938 . 
seul principe actif; le rôle des synergies entre composants et des interactions physiologiques dans l'obtention d'une action thérapeutique efficace; la prise en compte des relations entre corps et environnement non seulement pour intégrer la pathologie dans un contexte de vie mais aussi dans une perspective d'écologie botanique liant les propriétés d'une plante (en particulier les variations de composition dans le temps ou l'espace) et la dynamique des écosystèmes (les interactions entre espèces végétales). Cette version de la culture de la préparation a sans aucun doute été nourrie par les discussions des cercles de la médecine alternative allemande auxquels appartenait G. Madaus ainsi qu'à ses activités de publiciste, mais ses origines sont tout autant à chercher du côté des pratiques de production et de recherche interne établies dans les années 1920 et 1930. L'industrialisation des extraits de plantes telle que pratiquée au sein de Madaus a fait intervenir trois registres de pratiques : la mécanisation; la standardisation et le contrôle de qualité biologique; la mise en culture des plantes. À cela correspondent trois formes de savoirs élaborés dans un laboratoire de chimie, un institut de biologie et une ferme expérimentale. Ce dispositif technoscientifique liant de façon originale culture professionnelle de la préparation et industrialisation du médicament mérite description.

Tout d'abord, la mécanisation et la manipulation de la matière médicale par les machines. De façon tout à fait intéressante, il s'agit du seul domaine d'innovation pour lequel les frères Madaus jugèrent possible et utile de demander des brevets 9 . Ceux-ci ont en premier lieu porté sur une méthode qui revient dans toutes les publications et publicités comme le principal succès de la firme, c'est-à-dire la mise au point d'une méthode de fabrication de pilules à partir des plantes fraîchement collectées. Les préparations dites Teep (pour Tee-Pulver) permettaient en effet de transformer les poudres ou décoctions de plantes communes à toute la pharmacie galénique en véritables comprimés et dragées, mais sans en passer par l'isolement des principes actifs privilégié par la pharmacie chimique. Les annonces Madaus (fig. 1) proclament ainsi que les Teep conservent tous les éléments accompagnateurs, qu'elles n'éliminent ni les protéines, ni les hormones végétales, qu'elles sont de ce fait bien supérieures aux teintures et décoctions homéopathiques.

Telle que résumée (et idéalisée) par les experts de Madaus, la fabrication d'un Teep partait de la plante entière, ou du moins du matériel stocké immédiatement après la récolte, broyée en présence de sucre puis séchée partiellement à l'air chaud. Ce mélange, dit dilution 0 (par comparaison avec les décoctions homéopathiques), était ensuite «dilué » par mélange et pétrissage avec de nouvelles quantités de sucre pour obtenir le dosage de la plante tenu pour adéquat à la formulation. La machinerie utilisée pour faire des Teep était la même que celle rencontrée dans les firmes «classiques»: broyeurs, mélangeurs, dessiccateurs et surtout machines capables de compresser la poudre des mélanges pour fabriquer les comprimés. Le lien entre mécanisation et défense de la médecine biologique reposait sur une série continue d'expérimentations pratiquées au laboratoire de chimie et qui avait 


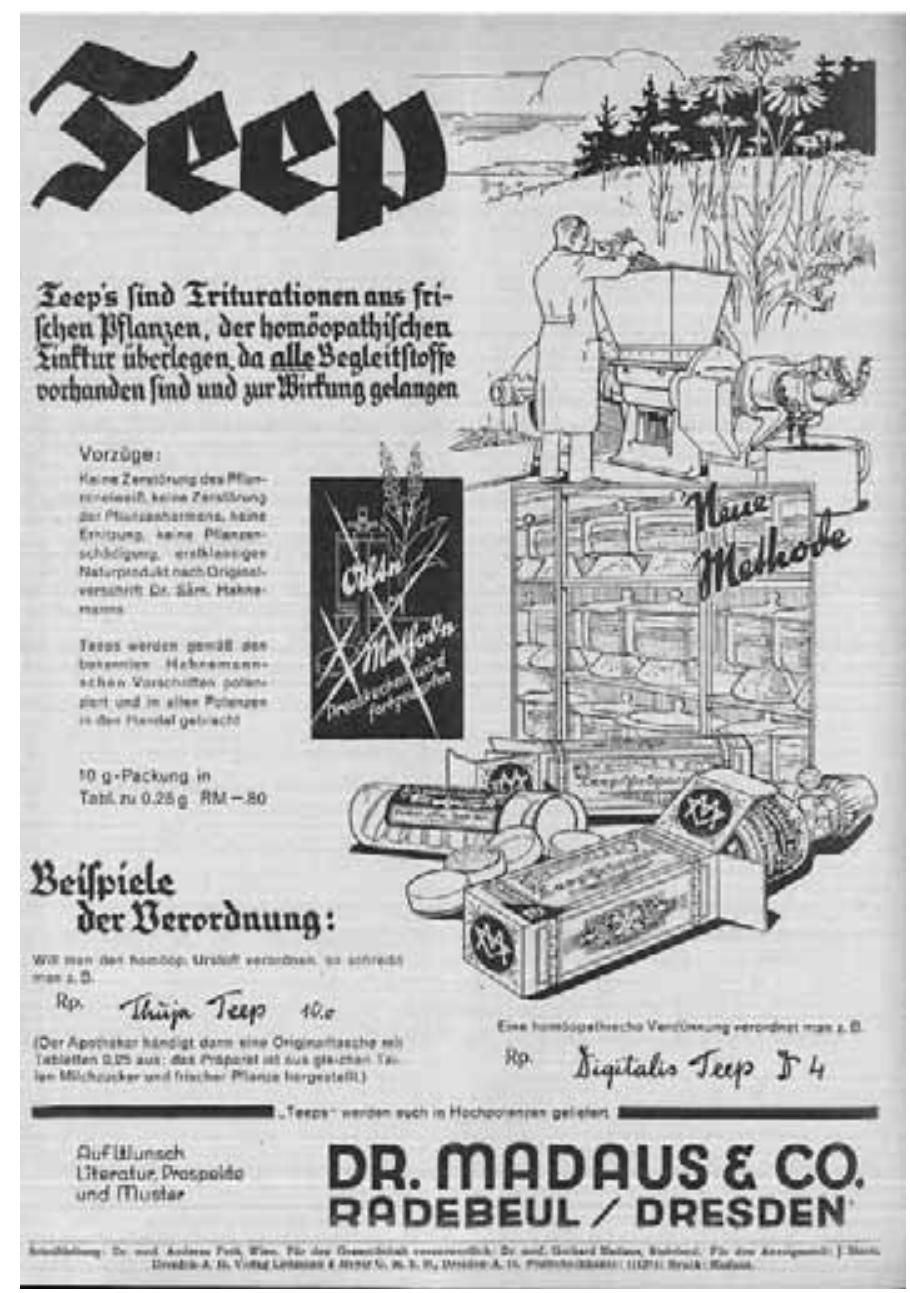

Figure 1. Vanter les mérites de la méthode Teep. Annonce parue dans Biologische Heilkunst, 1932. (C) Madaus AG.

pour objectif de démontrer la capacité de la procédure Teep à préserver le maximum de principes végétaux (actifs ou non) et de définir les protocoles d'élaboration des diverses formules commerciales (fig. 2) ${ }^{10}$.

Une seconde dimension du processus d'industrialisation des préparations poursuivi par Madaus concerne la surveillance des produits avec la multiplication des dispositifs de standardisation. Le phénomène n'est pas isolé et la pharmacie

10 - Voir par exemple, parmi des dizaines, les investigations réalisées en septembre 1937 avec les feuilles de belladone fraîchement récoltées et transformées en Teep avec du lactose, du fructose et du fructose additionné d'acide ascorbique : Sächsiche Staatsarchiv Dresden, 11610 Firma Dr Madaus, Aktenordner 127, Pharmazeutische Untersuchung Berichte. 


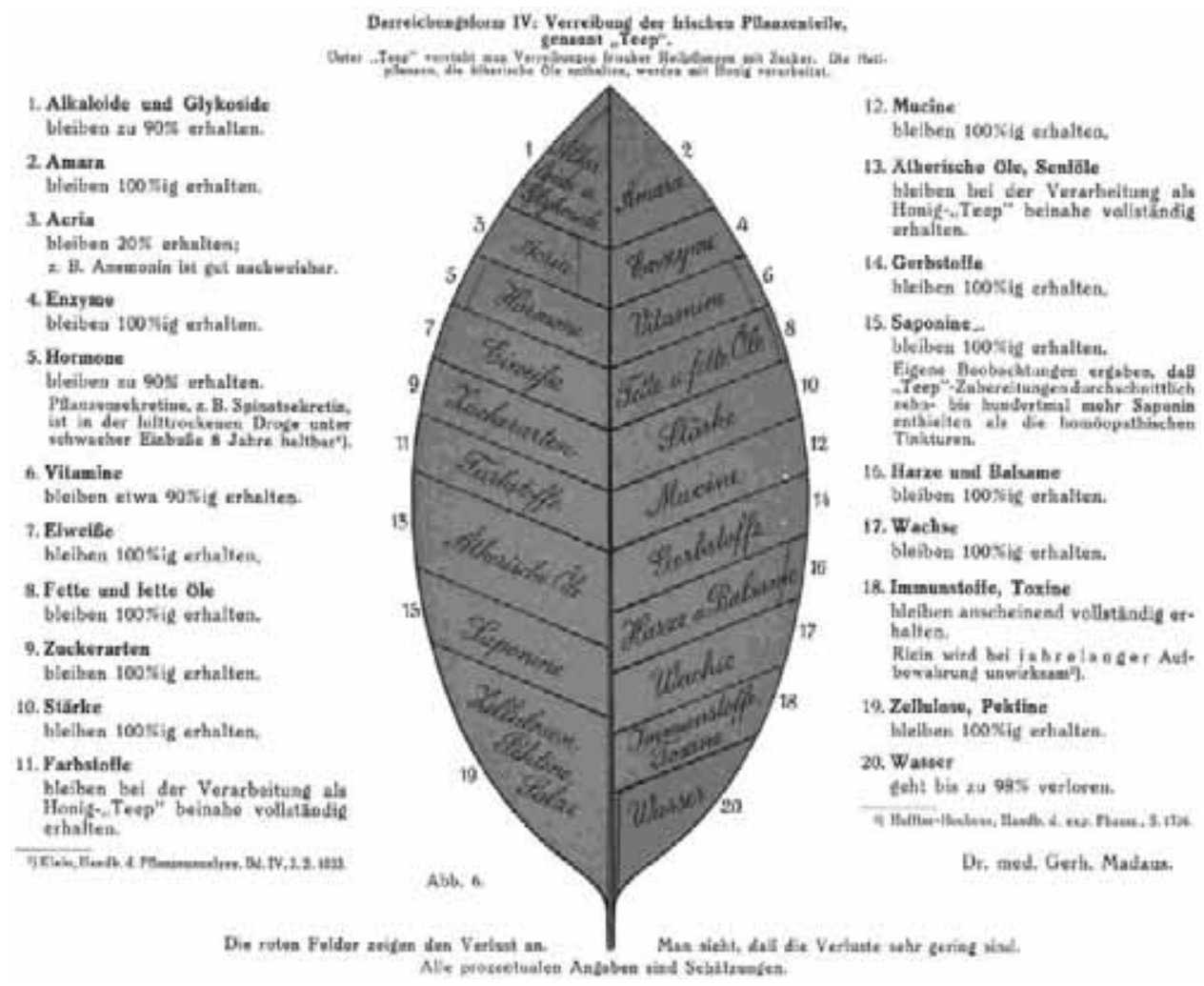

Figure 2. Préservation de la composition globale des végétaux par la procédure de préparation Teep. Dr. Madaus Jahrbuch, 1935. (C) Madaus $A G$.

du début du $\mathrm{XX}^{\mathrm{e}}$ siècle est obsédée par la standardisation. Les dispositifs auxquels renvoient ces discours sont d'une grande variété: nomenclatures, protocoles de travail, standards matériels, etc. ${ }^{11}$.

Madaus ne semble donc pas différente de ces nombreuses compagnies issues de l'officine qui exploitent la pharmacopée et les corpus de matière médicale pour y trouver les savoirs et recettes ouvrant la voie à la production de spécialités nouvelles dont les mérites proclamés tiennent autant à leur caractère prêt à l'emploi qu'à leur homogénéité certifiée. Ainsi le laboratoire de chimie de Madaus était en premier lieu un laboratoire d'essai et de contrôle de la qualité où une demidouzaine de personnes s'occupaient - sous la direction d'un pharmacien-chimiste Alfred Kuhn - de vérifier la composition des produits (en particulier en cas de réclamation), d'expérimenter des variantes aux protocoles de préparation, d'éprouver l'intérêt de tel ou tel test proposé dans la littérature chimique. Mais là encore, le renvoi à une approche «biologique» correspondait à des pratiques locales, aux

11 - Pour une discussion de ces pratiques de standardisation pharmaceutique, voir Christian Bonah, Christophe Masutti et Anne Rasmussen (dir.), Standard drugs and drug standards, à paraître. 
frontières de la pharmacologie, de la biochimie et de l'homéopathie. Les activités de contrôle de qualité et de standardisation du laboratoire de chimie sont, de ce point de vue, très intéressantes à analyser dans le détail. Deux exemples suffiront ici pour donner une idée de la différence avec un laboratoire de firme chimique, des liens étroits de cet univers avec la culture professionnelle de la préparation et des effets indéniables du passage à l'échelle industrielle.

Le premier exemple concerne les analyses de composition et leur utilisation à des fins de standardisation des Teep de belladone. Admettant que l'efficacité était, dans ce cas, due à la présence d'alcaloïdes, les chimistes de Madaus s'employèrent à trouver une combinaison d'étapes d'extraction et de mesures physiques pour quantifier en un seul essai les trois principaux alcaloïdes : l'atropine, l'hyoscyamine et la scopolamine ${ }^{12}$. La procédure fut ensuite systématiquement utilisée pour analyser les graines et racines de différentes variétés. La principale conclusion était que l'alcaloïde le plus efficace n'était pas l'atropine comme l'énonçait la littérature pharmaceutique, mais la scopolamine. Dans une perspective chimique, l'étape suivante aurait été la purification de cette dernière et l'introduction d'une nouvelle spécialité. L'alliance entre botanique, mécanique et physiologie déboucha au contraire sur une modification du protocole de préparation du Teep de belladone pour inclure un mélange de variétés et de parties des plantes de façon à préserver l'équilibre entre les trois alcaloïdes.

Le second point concerne la nature des essais pratiques menés pour évaluer la valeur des préparations, lesquels reposaient rarement sur les analyses de composition. Kuhn entreprit ainsi un travail de systématisation des évaluations du goût et de la couleur des préparations qui avaient une fonction discriminante, non seulement pour les herboristes, mais pour toute la pharmacie d'officine. L'industrialisation se traduisit dans ce cas par le recours à l'instrumentation physique, la formalisation des notations et la spécialisation du personnel entraîné à goûter et voir ${ }^{13}$. Les développements les plus importants ont toutefois porté sur l'usage des modèles animaux. Un exemple caractéristique est celui des extraits de digitale, pour lesquels les pharmacopées recommandaient l'utilisation d'un montage permettant l'isolement d'un cœur de grenouille et l'enregistrement de ses contractions comme moyen d'objectiver la valeur de stimulant cardiaque de la plante. Cherchant à économiser les grenouilles, le laboratoire de Madaus tenta de remplacer cet essai par un protocole basé sur l'observation de daphnies microscopiques dont les vacuoles pulsatiles sont sensibles aux extraits de digitale ${ }^{14}$.

La standardisation biologique a souvent été décrite comme une forme pragmatique et incomplète de standardisation moléculaire, une pratique rendue incontournable par l'absence de connaissances chimiques, appelée à être remplacée par des

12 - Sächsiche Staatsarchiv Dresden, 11610 Firma Dr Madaus, Aktenordner 127, Pharmazeutische Untersuchung Berichte, septembre 1939.

13 - Alfred KuHN, «Die Verteilung pflanzlicher Inhaltstoffe in Kapillarbild », Jahrbuch Madaus, 1935, p. 55-61.

14 - Alfred KuHN et H. PENZ, «Auswertungsversuche von Digitalis an Wasserflöchen », Jahrbuch Madaus, 1937, p. 74-78. 
tests de composition supposant la présence d'un principe actif essentiel, si ce n'est unique $^{15}$. Dans cette perspective, la recherche de relations linéaires entre doses et effets et l'homogénéisation des conditions matérielles de la manipulation sont à comprendre comme une forme imparfaite de test chimique puisque l'essai biologique met en équivalence l'intensité d'un effet physiologique ou cellulaire pris pour étalon de l'action thérapeutique et une unité conventionnelle traitée comme s'il s'agissait de la quantité d'effecteur présente dans la préparation. Cette lecture est, dans le cas de nombreux médicaments issus du vivant, téléologique dans la mesure où elle réduit une pratique de modélisation inscrivant les standards biologiques dans un processus global de cadrage physiologique des pratiques cliniques à une réduction moléculaire qui - parfois - n'interviendra que des décennies plus tard et dont la standardisation biologique a été une des conditions.

Mais ce dont il s'agit à Madaus est encore différent. En effet, contrairement aux mesures de «puissance» des sérums antidiphtériques, de l'insuline ou des hormones sexuelles dont il sera question plus loin et qui servaient à normaliser les doses que les fabricants conseillaient aux médecins d'injecter à partir d'unités physiologiques définies par les essais animaux (conduisant à l'adoption d'unité « cobaye», «lapin» ou «souris»), les étiquettes, emballages et notices des produits Madaus n'indiquaient pas les résultats d'essais biologiques, mais - comme les préparations homéopathiques - seulement la «dilution»du matériel végétal effectuée lors de la préparation. Les essais biologiques étaient pourtant essentiels : l'ampleur des investissements dans les laboratoires de chimie et de biologie tout autant que leur mise en avant dans les publications de la firme en témoignent. En plus de leur rôle de marqueurs de scientificité, ils avaient une valeur pratique interne.

Les dirigeants de Madaus partageaient avec la pharmacie de faculté une très grande fascination pour le laboratoire, estimant qu'en regard de la clinique il s'agissait du véritable site de formation des connaissances sur les médicaments. Cette hiérarchie se retrouve dans la façon dont les chercheurs de la firme entendaient «fouiller» le stock de connaissances de la médecine «populaire » pour découvrir de nouvelles indications et de nouvelles combinaisons. Le recours aux modèles animaux standards a ainsi non seulement servi à objectiver les effets biologiques des préparations complexes, mais aussi à gérer cette complexité dans une triple perspective : contrôler la variabilité des plantes collectées et des matières premières afin d'adapter les mélanges (et - si l'on suit le raisonnement sur la composition globale - d'assurer d'une certaine stabilité d'effets); surveiller le bon déroulement des protocoles (la question était ici au moins autant celle de leur validité en regard des conditions changeantes de production qu'une volonté de surveillance et de détection des erreurs des opérateurs); donner les moyens d'une réappropriation de l'expérience des effets des préparations issue du travail des médecins et thérapeutes.

Deux épisodes illustrent les rapports ambigus entre cette modélisation et les pratiques de ces derniers. Le premier concerne l'utilisation du gui (Viscum albu) vendu 
sous forme de poudre et de Teep, auquel la littérature pharmaceutique attribuait une valeur antispasmodique (justifiant une utilisation dans les cas d'épilepsie et de maladies nerveuses) et un effet hypotenseur exploité dans les situations de trop forte pression, d'athérosclérose, de migraine, à quoi s'ajoutaient dans certains écrits de médecine populaire (en particulier ceux des praticiens anthroposophes) des effets anticancéreux ${ }^{16}$. S'appuyant sur la modélisation des effets des extraits de gui sur le système circulatoire réalisée par le laboratoire de matière médicale de la faculté de pharmacie de Paris, Madaus privilégiait leur emploi contre l'hypertension et l'athérosclérose. Les autres usages n'étaient toutefois pas éliminés. Une des spécificités des pratiques de recherche internes à Madaus était ainsi d'associer physiologie végétale et physiologie animale. L'enjeu des premières était non seulement de disposer de savoirs sur les plantes, leur croissance et leur composition, mais aussi d'exploiter l'unité du monde vivant, les correspondances entre effecteurs biologiques et les analogies entre les systèmes fonctionnels végétaux et animaux. Les expériences avec les extraits de gui menées en serre et sur les cultures de végétaux étaient présentées dans l'almanach Madaus comme la preuve de leur action comme hormone capable d'empêcher la cicatrisation en bloquant la division des cellules au niveau des tissus blessés, une propriété susceptible d'expliquer non seulement le parasitisme naturel du gui mais aussi sa capacité éventuelle à entraver la croissance des cancers ${ }^{17}$. L'acceptation de cette correspondance n'alla toutefois jamais au-delà de l'expérimentation interne, par exemple jusqu'à la promotion d'une indication du Teep de gui comme anticancéreux.

Le second exemple est celui des recherches sur l'ail (Allium sativum), une plante figurant en bonne place dans le catalogue de Madaus, vendue sous la forme de pilules Alliocaps dont les extraits étaient mentionnés dans la plupart des pharmacopées européennes en relation avec le traitement des rhumatismes, des problèmes articulaires et de l'athérosclérose. Le rôle du laboratoire de biologie Madaus n'a pas été de chercher de nouvelles indications, mais de renforcer ces usages avec la mise au point d'un modèle animal de la maladie dans lequel une athérosclérose était provoquée chez des souris par administration prolongée de quantités importantes de vitamine $\mathrm{D}$ et contrée par l'ingestion parallèle d'extraits d'ail ${ }^{18}$.

Plus généralement, hormones et vitamines, ces emblèmes des nouveaux savoirs biologiques et tout particulièrement de la biochimie d'entre-deux-guerres, occupent une place tout à fait particulière dans les recherches et la littérature scientifico-publicitaire de Madaus. Les hormones sexuelles avaient ainsi un double statut. D'une part, G. Madaus prenait pour cible leur usage croissant en gynécologie au nom de cette idée «simpliste» selon laquelle on pourrait les prescrire dans des

16 - G. Madaus, Lehrbuch der biologischen Heilmittel, op. cit., vol. III, p. 2833-2840; Id., «Wissenchaftlichen Grundlage der Signaturen Lehre », Biologische Heilkunst, 13, 1932, p. 649; Id., «Versuche über Wundheilung bei Pflanzen», Biologische Heilkunst, 14, 1933 , p. 332.

17 - Ernst KocH, «Biologische Untersuchungen ", Jahrbuch Madaus, 1936, p. 42-58.

18 - G. Madaus, Lehrbuch der biologischen Heilmittel, op. cit., vol. I, p. 473 ; E. КосH, «Biologische Untersuchungen», art. cit. 
cas de désordres de la menstruation ou - pire - de situations problématiques comme la ménopause parce qu'il s'agirait de maladies de «carence» en hormones féminines ${ }^{19}$. D'autre part, ces mêmes substances étaient intégrées au monde de la médecine biologique alternative, présentées comme de très puissants effecteurs physiologiques communs aux plantes et aux animaux. Cette communauté expliquait que - comme les biologistes de la firme l'avaient démontré - des hormones sexuelles animales puissent agir sur la croissance ou la différenciation des végétaux, mais surtout que certaines plantes, notamment celles utilisées de longue date contre les «maladies des femmes» comme le romarin ou la pulsatile, contiennent des hormones agissant sur la physiologie sexuelle humaine. En conséquence, une hybridation passant par un examen des préparations traditionnelles sur les systèmes expérimentaux de la biochimie des stérö̈des était non seulement possible, mais indispensable à la modernisation industrielle ${ }^{20}$.

L'accent mis sur l'origine naturelle des substances, sur leur rôle régulateur, sur la diversité de leurs effets, sur leur action à très faible dose (leur action catalytique) est un discours partagé par les biologistes de Madaus, par les pharmaciens de l'université de Berlin ainsi que par les biochimistes de la Kaiser Wilhelm Gesellshaft. Cet intérêt pour les «biologiques» était suffisamment fort pour que ceux-ci fassent non seulement fonction d'objets-frontières entre le monde de la matière médicale et celui de la spécialité industrielle, mais aussi pour qu'ils opèrent comme instrument de traduction de la préparation en purification. Pendant la guerre, Madaus entrepris par exemple une longue série d'investigations sur les vitamines et la possibilité d'utiliser certains conifères poussant communément en Allemagne comme sources alternatives d'extraits riches en vitamine $\mathrm{B}$ et $\mathrm{C}^{21}$. Dans le cas de la vitamine $\mathrm{C}$, qui occupa une part importante des activités du laboratoire de chimie entre 1940 et 1942, si les écrits de Kuhn mettaient bien en avant le caractère naturel des préparations, le discours sur les synergies avait purement et simplement disparu de sorte qu'en pratique les tests de composition utilisés pour identifier les plantes candidates se résumèrent aux essais chimiques de l'acide ascorbique. Le choix initial des aiguilles de sapins et d'épicéas comme matière première ne reposait que sur ce seul critère moléculaire. Après plusieurs mois de travail sur la composition, cette piste fut toutefois abandonnée. Les motifs de cette décision n'avaient rien à voir avec ce « réductionnisme » et avec l'importance des associations, mais invoquaient seulement le fait que le goût des extraits était si amer qu'il rendait illusoire la création d'un marché. Il reste toutefois vrai qu'à aucun moment Kuhn ou Madaus n'envisagèrent de remplacer la préparation de décoction ou de Teep par une procédure de purification complète qui aurait conduit

19 - G. MADAUS, « Biologische Forschung und biologische Therapie », Biologische Heilkunst, 14, 1933, 289-291.

20 - Gerhard Madaus et Arndt KunZe, «Einige Ergebnisse der Untersuchungen in unserer biologischen Versuchstation im Jahr 1932 », Jahrbuch Madaus, 1933, p. 11-24. 
à des comprimés de vitamine $\mathrm{C}$ comparables à ceux que la firme suisse Roche introduisait au même moment sur le marché allemand ${ }^{22}$.

Une troisième dimension de l'industrialisation des préparations concerne la mise en culture de certaines plantes médicinales. La conduite et le rendement de la collecte annuelle étaient des problèmes récurrents, suscitant des inquiétudes dès le début de la saison, au printemps, et aboutissant fréquemment à un constat de pénurie, une fois les plantes rentrées en fin d'été ou début d'automne ${ }^{23}$. La solution rêvée par la direction de Madaus était la mise en culture et l'abandon des prélèvements en prairies et forêts. Pour quelques espèces comme la digitale, la domestication avait réussi mais le processus était exceptionnel et non sans susciter des réserves quant à ses effets sur la vie, la physiologie, la composition et du coup l'efficacité des extraits de ces plantes cultivées. Ainsi, la belladone, une fois mise en culture et stimulée par l'utilisation d'engrais, se trouvait-elle - pour les responsables de Madaus - plus toxique qu'à l'état sauvage ${ }^{24}$.

Une part significative du travail du laboratoire de chimie et de la ferme expérimentale portait donc sur l'exploration des conditions qui pourraient rendre possible cette mise en culture. Les comptes rendus d'essais révèlent deux types de questionnement. Une première série d'expériences avait pour ambition de mettre en évidence les effets des variations des conditions de culture (par exemple de la nature des fertilisants) sur la composition et les propriétés thérapeutiques des extraits (les deux n'étant pas déductibles l'un de l'autre). Par exemple, la belladone cultivée sur des sols sableux semblait beaucoup plus riche en huiles essentielles (extraites à l'éther) que celle cultivée sur sols argileux ${ }^{25}$. Ce constat avait des conséquences non seulement sur ce qu'on faisait à la ferme mais aussi (même si nous n'en avons pas beaucoup d'exemples) sur l'optimisation des mélanges.

Une seconde série d'expérimentations portait sur les associations entre végétaux. La botanique de Madaus était une botanique collectiviste approchant les plantes non pas comme des organismes isolés mais comme des espèces intégrées à des communautés relativement stables. Madaus entendait donner un caractère scientifique à l'importante littérature sur les antipathies et sympathies entre plantes qui faisait le lien entre botanique populaire et écologie académique. Sans atteindre la complexité de la notion alors émergente d'écosystème, ces associations entre plantes compagnes étaient, pour les phytosociologies que Madaus aimait citer, des regroupements hiérarchisés et caractéristiques d'un milieu de vie. Qu'il s'agisse d'échanges matériels directs ou de changements des sols, la nature des communautés végétales affectait la physiologie et donc la composition des espèces collectées. Trouver un bon système de culture supposait donc de reproduire, sous une forme stabilisée et raisonnablement simplifiée, les relations les plus importantes entre 
une variété médicinale et certaines de ses compagnes. Encore fallait-il pouvoir les définir et donc les rendre visibles, en particulier étudier la diffusion de produits sécrétés par les racines qui était, pour Madaus, un facteur explicatif essentiel donnant une identité matérielle aux influences que l'analyse statistique des populations ne faisait que postuler ${ }^{26}$.

Il n'existe que peu de vestiges des essais réalisés à la ferme, seuls ceux conduits dans le laboratoire de biologie ont laissé des traces dans les archives. Ils portaient sur des combinaisons d'espèces prises deux à deux. Dans le cas de la violette (utilisée dans le traitement des maladies de peau, de l'eczéma en particulier), l'association avec deux céréales (le blé et l'orge) donnait des résultats radicalement différents, l'orge stimulant la germination des violettes tandis que le blé l'inhibait ${ }^{27}$. La belladone est le seul exemple connu où ces explorations ont débouché sur un nouveau protocole de culture. Cultivée avec une légumineuse officinale (Galega officinalis - faux indigo ou sainfoin d'Espagne) (prescrite contre le diabète) la digitale se montrait nettement plus riche en alcaloïdes ${ }^{28}$. Un plan d'exploration systématique de ces associations fut proposé en 1937 mais il ne semble pas avoir été mis en œuvre. De plus, la littérature de la firme touchant aux conditions de culture privilégie - comme les agronomes et chimistes agricoles - la question de la fertilisation, des engrais et des apports de minéraux ${ }^{29}$. Enfin, il semble que les cultures en champ aient été suffisamment difficiles à mettre au point pour que la majorité d'entre elles ait été réalisée sans relation aucune avec cette éco-physiologie holiste, dans les conditions moins exigeantes et mieux contrôlées des serres de production. Ce fut par exemple le cas d'une espèce de cactus (Cactus grandifolia) utilisée dans une préparation contre l'angine de poitrine (Goldtropfen). La version importée de Cactus grandifolia fut remplacée par une culture locale dont les centaines de pots alignés symbolisaient la capacité d'innovation botanique de la firme.

Avant de refermer le chapitre Madaus, il reste à évoquer une dernière sphère de savoirs qui a contribué à l'industrialisation des plantes médicinales. Celle-ci n'avait pas de lien direct avec la manipulation de la matière médicale, mais concernait les relations avec les «clients». Dans les années 1930, la firme de Dresde juxtaposa deux manières d'organiser la «propagande» pour ses remèdes prêts à l'emploi. La première - ancienne - centrée sur les annonces et la réputation du pharmacien producteur, dans ce cas de la firme, mettait en avant la marque et prenait pour cible à part égale médecins et patients. La seconde manière était la «propagande» scientifique en direction des médecins et des thérapeutes dont l'importance a crû à raison des investissements de scientificité. Faire circuler auprès des médecins une documentation mêlant encarts publicitaires et articles scientifiques incluant résultats d'expérimentation émanant des compagnies, jugements

26 - G. MaDaus, Lehrbuch der biologischen Heilmittel, op. cit., vol. I, p. 78-79.

27 - Gerhard MADAUS, « Die Wirkung von Digitalis purpurea und ihrer Abhängigkeit von der Düngung », Jahrbuch Madaus, 1935, p. 21.

28 - Sur Galega, voir G. Madaus, Lehrbuch der biologischen Heilmittel, op. cit., p. 1407; sur 
de faits et recommandations pour la pratique est devenu, dans l'entre-deux-guerres, une dimension centrale de la construction du marché des spécialités. La propagande scientifique mise en place par Madaus à partir de 1925 a privilégié trois supports : les luxueux almanachs déjà cités; la publication de manuels et de compendia; le soutien à des journaux comme Biologische Heilkunst dans lequel G. Madaus ou ses collaborateurs scientifiques publièrent de nombreux articles et où la firme insérait chaque mois deux ou trois annonces. Ce qui était en jeu n'est pas le fait que les médecins prescrivent et agissent en «gardiens» de l'accès au médicament: la prescription n'était obligatoire que pour une petite minorité des préparations de plantes. La cible était la fonction d'intermédiaire des professionnels qu'il s'agisse ou non de médecins, leur travail d'expertise et donc l'articulation entre deux objectifs difficilement compatibles: d'une part formater le jugement des praticiens pour faire accepter les usages imaginés par le fabricant, et d'autre part faire remonter les savoir-faire thérapeutiques pour les intégrer aux choix d'innovation et de commercialisation.

Parmi les nouveaux outils publicitaires, le très systématique compendium Madaus des médicaments biologiques (Lehrbuch der biologischen Heilmittel) est intéressant à considérer du fait de la proximité qu'il entretient avec une pharmacopée puisqu'il incluait plus de 400 plantes décrites au long de près de 3000 pages. Pour chaque plante prise en considération, les informations portaient toujours sur les dénominations, l'aire de répartition naturelle (avec cartes à l'appui), la description des différentes parties de la plante, l'identification des matières utilisées et de leur mode de conservation, l'histoire des usages. La suite de la description était en tous points conforme à la tradition professionnelle et pharmacologique, listant les effets rapportés dans la littérature (définie de façon plus large que dans les pharmacopées), les discussions sur la nature des substances actives, les tests éventuels de composition, avant d'aborder les recommandations sur les parties à utiliser, les doses et les associations (recettes) couramment pratiquées en privilégiant une présentation par indications ou catégories de symptômes.

Si l'on prend l'exemple de la belladone, le codex Madaus inclut ainsi tout ce que l'on pouvait trouver dans la pharmacopée officielle, mais avec de nombreuses indications originales sur la plante, l'histoire de ses usages (ses mentions dans les ouvrages de Discorides, Gesner ou Albert le Grand, Boerhave) et surtout la variété de ses emplois thérapeutiques ${ }^{30}$. L'évaluation des diverses «cures» est dominée par l'intérêt pour les combinaisons et non pour les effets attribuables à la seule atropine (comme dans la pharmacopée allemande). Le codex Madaus discute ainsi longuement une cure dite bulgare, un remède très utilisé en Italie dont la formule restée secrète - ce que critiquait fortement G. Madaus - incluait une racine, une poudre noire, une pilule et des fragments d'une seconde racine; le tout à utiliser selon un protocole très strict. Des travaux réalisés par divers instituts et pharmaciens académiques qui s'y étaient intéressés avant Madaus convergeaient vers un mélange de belladone, de charbon animal, de noix de muscade et de racines d'Acorus calamus. 
En continuité avec les analyses de laboratoire évoquée plus haut, toute la discussion de l'efficacité de cette cure dans le traitement de l'épilepsie, des tremblements et des crampes était ramenée à la quantité globale d'atropine et d'alcaloïdes de belladone ainsi ingérée. La partie pharmacologique de l'article belladone ne se distingue en rien de ce que l'on peut trouver dans le Deutsches Ar:neibuch, y compris lorsqu'il s'agit des recommandations de doses «homéopathiques» (D3 à D5) qui étaient en fait, comme souvent avant que les hautes dilutions deviennent une norme de la pratique homéopathique, équivalentes à celles de la pharmacopée officielle.

Dans le compendium Madaus, le marqueur le plus original de cet intérêt pour les thérapies locales et alternatives est finalement l'utilisation des résultats d'une grande enquête réalisée au milieu des années 1930 sur les usages des préparations végétales. Ayant contacté des milliers de médecins et thérapeutes officiellement enregistrés, la firme réussit à analyser près de 12000 questionnaires listant les plantes utilisées régulièrement ainsi que les motifs ou symptômes à l'origine de leur prescription ${ }^{31}$. G. Madaus s'en servit ensuite pour calculer des fréquences d'emploi par maladie ou par espèce incorporées sous forme de courbes d'utilisation des préparations présentées dans le compendium et qui constituent un nouvel indice de combinaison entre régulation professionnelle et régulation industrielle du médicament, centrée, dans ce cas, sur l'autonomie des thérapeutes, l'apprentissage par la routine clinique et la mobilisation commerciale de cette expertise.

\section{Schering, la régulation industrielle et la molécularisation des hormones sexuelles}

Durant les mêmes années, la firme berlinoise Schering AG était grande productrice d'un autre type d'extraits provenant en l'occurrence non plus des plantes mais des glandes endocrines animales (préparations dites opothérapeutiques). Schering était même en position dominante pour tout ce qui touchait aux préparations d'hormones sexuelles. La firme avait non seulement été directement associée à l'isolement et à la caractérisation chimique de ces substances, mais ses trois marques Progynon, Proluton, Testoviron étaient, en Allemagne, les formes les plus vendues d'agents thérapeutiques contenant des stéroïdes sexuels mâles ou femelles. Dans son travail d'invention de ces spécialités, Schering avait suivi une stratégie alternative à celle adoptée par Madaus, transformant les préparations opothérapeutiques du début du siècle en molécules pures et, pour certaines d'entre elles, modifiées par la chimie organique. Pourtant, si la trajectoire de Schering illustre bien une configuration d'industrialisation de la préparation où biochimie et manipulation des molécules ont le beau rôle, les parentés avec les activités de Madaus sont significatives et renvoient à la montée des pratiques de régulation industrielle du médicament.

Contrairement à celle de Madaus, l'histoire de l'entreprise Schering est relativement bien connue: les origines de la firme, ses stratégies de développement, 
sa position économique ou sa place sur le marché international des produits chimiques et pharmaceutiques ont fait l'objet de travaux historiques ${ }^{32}$. Du fait de sa taille et de son rôle dans la production et la commercialisation de produits chimiques de base comme le camphre, la firme de Berlin a souvent été associée à Bayer, Hoechst et aux firmes constitutives du trust IG Farben ${ }^{33}$. La conséquence d'un tel rapprochement est d'admettre que Schering a la même histoire que les firmes productrices de colorants, que - dotée de fortes capacités de travail en chimie organique elle a progressivement investi le marché pharmaceutique en adoptant le screening (criblage) chimico-pharmacologique comme modèle d'innovation ${ }^{34}$. Cette comparaison est source de confusion parce qu'elle suppose qu'il y aurait eu une seule (bonne) manière de «moléculariser » les agents thérapeutiques et surtout parce que Schering n'a adopté le screening - cette conjonction entre la synthèse par les chimistes organiciens de centaines de molécules analogues et le test systématique de leurs propriétés sur des modèles animaux de maladie-comme mode d'organisation de la recherche que dans les années 1960, dans un tout autre contexte de sorte que, jusqu'à la Seconde Guerre mondiale, savoirs et préparations biologiques dominent la recherche interne même si les hormones sexuelles sont restées, jusqu'à l'introduction de la pilule contraceptive, un marché de niche avec des marges importantes mais des volumes de ventes modestes.

Le paradoxe de la forme d'industrialisation du médicament mise en œuvre à Berlin est en effet d'avoir progressivement marginalisé la production chimique. Schering est bien une compagnie fondée par un pharmacien, mais ce n'est pas une firme issue de l'officine. Tout au long du XIXe siècle, la compagnie a produit une vaste palette de composés (elle en compte près de 800 à son catalogue en 1890), mais ceux-ci comprenaient très peu de spécialités pharmaceutiques. La grande majorité relevait de ces produits intermédiaires utilisés par les pharmaciens pour réaliser leurs préparations et qui ont progressivement été sortis des pharmacopées car considérés comme des matières premières indispensables mais dont la production ne relevait plus des compétences spécifiques du pharmacien et donc de ce que la profession devait réguler. La façon dont Schering s'est initialement insérée dans l'ordre professionnel avait consisté à vendre en grandes quantités et avec une qualité (dans ce cas identifiée à la pureté) certifiée, non des spécialités ou des remèdes secrets, mais l'infrastructure chimique du travail d'officine ${ }^{35}$.

Entre 1900 et 1920, ce positionnement a trouvé ses limites du fait d'une compétition accrue avec les grandes firmes de la chimie et de l'érosion continue des prix des produits en vrac. Schering aurait alors pu devenir l'une des branches

\footnotetext{
32 - W. Wimmer, «Wir haben fast immer was Neues»..., op. cit.; Hans Höllander, Geschichte der Schering Aktiengesellschaft, Berlin, Blaschker, 1955; Christopher KoBRACK, National cultures and international competition: The experience of Schering AG, 1851-1950, Cambridge, Cambridge University Press, 2002.

33 - Voir W. Wimmer, «Wir haben fast immer was Neues»..., op. cit.

34 - Sur l'histoire du screening chez Bayer, outre J. LESCH, The first miracle drug..., op. cit., voir Jean-Paul GaudilliÈre, «Die Geburt des Screenings », Medizinisches Historiches Journal, sous presse.

35 - Sur ce point, voir C. KobRack, National cultures and international competition..., op. cit.
} 
d'IG Farben ou s'intégrer à un autre cartel de la chimie européenne. La variété de ses productions tout autant que la diversité des savoirs « maison » ont rendu possible une alternative «biologique». En effet, paradoxalement, Schering employait peu de spécialistes de la synthèse organique. Ses chimistes étaient nombreux, mais étaient en premier lieu des experts de la production et du contrôle de qualité. Jusqu'à la Première Guerre mondiale, en un écho inattendu à la culture des pharmacopées, l'enjeu des investigations menées en interne était moins de trouver de nouvelles molécules que d'améliorer ou de modifier les protocoles de fabrication de celles qui étaient déjà connues ${ }^{36}$. Un des effets de cet usage instrumental de la chimie a été de laisser place à d'autres sources d'innovation. Un des premiers laboratoires internes, qui ne soit pas seulement un service de test mais aussi un site expérimental, a ainsi été un laboratoire de physiologie, inauguré en 1902.

Dans les années 1920, la combinaison de recherche physiologique et de contrôle de qualité des préparations que l'on y pratiquait entra en résonance avec les modifications du marché et le succès des thérapies hormonales auprès des gynécologues et d'une large gamme de médecins incluant dermatologues, psychiatres ou spécialistes de médecine interne ${ }^{37}$. S'enclenche ainsi un cercle «vertueux » liant investissements croissants de recherche biologique, molécularisation des extraits et production de masse de médicaments hormonaux. En une quinzaine d'années (1925-1940), Schering se trouva ainsi passer d'une vente financièrement seconde d'extraits d'organes (pancréas, testicules, ovaires) à la distribution massive et beaucoup plus lucrative de molécules purifiées (insuline, testostérone, œstrogènes et progestérone) avec, en perspective, la mise au point d'une synthèse partielle qui permettrait de résoudre le problème de la collecte laborieuse des matières premières, qu'il s'agisse des organes prélevés dans les abattoirs ou, à partir de la fin des années 1920, de la collecte dans les fermes des environs de Berlin d'urine de jument gravide spécifiquement utilisée pour la purification des œstrogènes.

En décembre 1938, Walter Schoeller, alors directeur du Hauptlaboratorium, le laboratoire issu de la croissance des activités de recherche interne, participait à une conférence du département «médical et scientifique » destinée à former les responsables des relations avec pharmaciens et médecins au sein des représentations locales de Schering. Il y présenta un rapport d'ensemble sur l'état des recherches sur les hormones et sur la contribution propre de la firme ${ }^{38}$. Le texte était pour l'essentiel consacré à quatre produits du catalogue: les trois hormones sexuelles (folliculine, hormone du corps jaune et testostérone) et la cortine, hormone des glandes surrénales. Une table chronologique rappelait les principales étapes de ce que Schoeller considérait comme un changement décisif des connaissances sur la physiologie et la nature des hormones, des produits et des protocoles de préparation.

36 - H. Höllander, Geschichte der Schering Aktiengesellschaft, op. cit.; W. WIMMER, «Wir haben fast immer was Neues "..., op. cit.

37 - Sur la gynécologie, voir Martina SCHLuender, «Reproduktionen, Experimentalisierung der Geburtshilfe zwischen 1900 und 1930 », thèse de médecine, Berlin, 2007. 38 - Archives Schering, Bestand 02, Ordner 1585 : W. Schoeller, Tagung der medizinische wissenschaftliche Abteilung, décembre 1938. 
La trajectoire des trois types d'hormones sexuelles vendues par Schering à partir de la fin des années 1920 est particulièrement révélatrice de la façon dont la production des extraits thérapeutiques a été intimement liée à la molécularisation des substances physiologiquement actives, laquelle a en retour rendu plus crédible les investissements dans le secteur des «biologiques ${ }^{39}$. Un attrait particulier des nouvelles hormones tenait en effet aux modalités de leur inclusion dans l'arsenal thérapeutique : par la mise au point de protocoles de préparation assimilés non à de la pharmacie mais à des pratiques de biochimie industrielle justiciables d'un statut propriétaire garanti par une combinaison de secret ou de brevets de procédés. Pour rendre compte de cette dynamique, il faut, comme dans le cas de Madaus, s'intéresser au travail scientifique interne, aux collaborations qu'il a suscitées et à la façon dont il a été utilisé pour la construction du marché des hormones.

Chez Schering comme chez Madaus, industrialisation rime avec standardisation, de sorte que l'on y retrouve l'utilisation multiforme des essais biologiques, les investissements dans la mise au point de procédures locales pour rendre visibles les propriétés des extraits, mais avec cette spécificité qu'il s'agissait de l'action féminisante ou masculinisante et donc tout autant de sexualiser les préparations et les substances que de quantifier leurs effets ${ }^{40}$. Dans ce cas toutefois, culte de la précision et mesure quantitative de la puissance sont omniprésents, indissociables de la culture de la pureté transposée par Schering des produits pharmaceutiques intermédiaires aux hormones et préparations biologiques. La différence avec la situation hybride de Madaus tient à la systématisation et à l'échelle des pratiques de standardisation.

Le laboratoire central est ainsi devenu un lieu où l'on s'occupait d'élever souris, lapins et rats ${ }^{41}$. On y préparait les animaux pour les expériences avant d'injecter les entités à tester et de suivre, jour après jour, les effets toxiques et physiologiques des extraits. Il s'agissait de vérifier la bonne qualité des matières premières ou la bonne conduite de la production, mais surtout d'obtenir ces nombres garants de la pureté (éprouvée biologiquement) et de la concentration des extraits et qui figuraient en bonne place sur chaque lot d'hormones avec les indications de doses à utiliser. Schering embaucha ainsi en 1925 un pharmacologue, Karl Junkmann, dont les activités incluaient recherche et contrôle de qualité d'une part importante du catalogue Schering: dérivés de l'arsenic, sulfonamides, adrénaline, strychnine, thymol, préparations hépatiques anti-anémiques, hormones de la thyroïde, etc. Junkmann était ainsi responsable des tests réalisés sur les conteneurs d'urine de jument qui arrivaient chaque semaine dans les locaux de l'usine berlinoise. L'utilisation ou non de la collecte dépendait en effet du résultat d'un test d'inoculation

39 - Pour la cortine, un développement similaire intervient après 1945, en parallèle avec celui de la cortisone par Merck.

40 - Sur ce point, voir Nelly Oudshoorn, «On measuring sex hormones: The role of biological essays in sexualizing chemical substances ", Bulletin of the History of Medicine, 64-2, 1990, p. 214-238.

41 - Walter HoHLwEG, « Biologische Arbeiten im chemischen Laboratorium », Medizinische Mitteilungen, 10, 1938, p. 3-8. 
à des souris systématiquement réalisé pour vérifier l'existence, dans l'urine-matière première, d'une activité œstrogène (repérée par les changements d'apparence des cellules vaginales) ${ }^{42}$.

Dans ce contexte, les protocoles d'essais étaient des résultats en soi, justiciables de publication comme pour ce test dit «à la crête de coq » utilisé pour évaluer les préparations de testostérone et autres substances masculinisantes (fig. 3):

Des poulets de la race Leghorn blanche sont castrés à l'âge de 8-10 semaines. Des mesures répétées de la surface de la crête doivent garantir qu'au cours des 8-10 semaines suivant la castration ce caractère sexuel secondaire reste stable. Seuls les animaux présentant une telle stabilité doivent être utilisés pour les expériences. La première phase est l'inoculation d'une solution contrôle contenant une quantité connue d'hormone pour vérifier la capacité de réponse de l'animal. Les animaux qui ne réagissent pas normalement ou dont la crête n'est pas revenue à sa taille initiale à la fin du test doivent être écartés. [...] La mesure de la puissance des hormones [mâles] consiste en une mesure de la croissance de la crête selon le protocole suivant. Des photographies de la crête sont prises avant l'inoculation d'épreuve puis à nouveau après 3 et 4 jours. Pour réaliser ces photographies, on place la crête sur une feuille de papier photosensible. On éclaire brièvement et on révèle. La surface de la crête est mesurée à l'aide d'un planimètre. Une unité poulet est définie comme la quantité présente dans une solution de $1 \mathrm{~cm}^{3}$ qui provoque en moyenne dans un intervalle de deux jours un accroissement de $20 \%$ de la surface de la crête, la mesure étant réalisée chez trois oiseaux différents ${ }^{43}$.

Une seconde particularité du dispositif technoscientifique de Schering par rapport à celui de Madaus est que, comme dans les grandes entreprises de l'électricité et de la chimie non pharmaceutique, l'autonomisation et la diversification des laboratoires internes ont favorisé une «fondamentalisation» des investigations ${ }^{44}$. À la fin des années 1920, les laboratoires de physiologie et de pharmacologie de Schering se sont ainsi engagés dans une exploration des effets des hormones sexuelles dont l'enjeu n'était plus l'identification des substances mais la compréhension des mécanismes de la reproduction sexuelle, les fonctions normales et modes d'action des hormones, leur implication dans les processus pathologiques. Jouissant d'un accès

42 - Sur la standardisation industrielle chez Schering, voir Jean-Paul GAUdiLLIÈrE, "The visible industrialist: Standards and the manufacture of sex hormones", in C. GRADMANN et J.SIMON (dir.), Evaluating and standardizing therapeutic agents 18901950, Londres, Routledge, 2010, sous presse.

43 - Adolf ButenANDT et Kurt TsCHERNING, «Über Androsteron, ein krystallisiertes männliches Sexualhormon », Hoppe-Seyler's Zeitschrift für physiologische Chemie, 299-4/6, 1934, p. 167-184.

44 - Dans les années 1920, la croissance des investissements de recherche s'est traduite non seulement par celle des effectifs du Hauptlaboratorium (avec un doublement de 30 à 60 du nombre des employés, dont la moitié de collaborateurs scientifiques), mais aussi par la création de deux divisions d'expérimentation biologique avec un laboratoire de physiologie (dirigé par W. Holweg) et un laboratoire de pharmacologie (sous la responsabilité de K. Junkmann). W. Wimmer, «Wir haben fast immer was Neues »..., op. cit. 


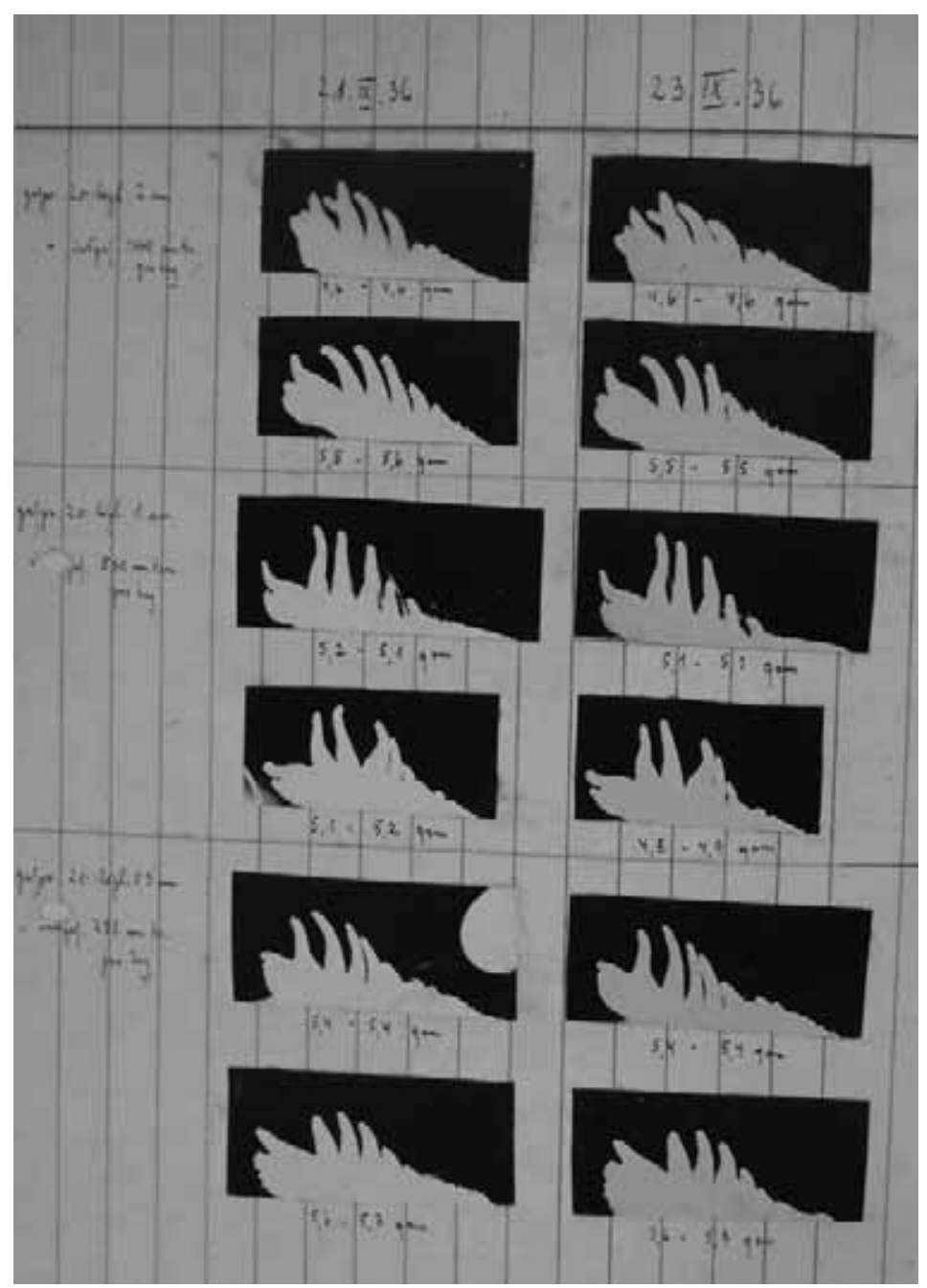

Figure 3. Le test à la crête de coq comme outil de contrôle de qualité. Schering, 1936. @ Bayer-Schering AG.

quasi illimité aux œestrogènes, alors rares et chers, les chercheurs de Schering furent ainsi les premiers à proposer un modèle de régulation du cycle sexuel faisant intervenir le cerveau, la glande hypophysaire et les ovaires, dans lequel les fortes concentrations en œstrogènes sanguins ont un effet « en retour » sur l'hypophyse, inhibant la sécrétion des facteurs stimulant l'activité et la maturation ovarienne ${ }^{45}$.

45 - Sur ces travaux et la dimension industrielle de l'étude des régulations, voir JeanPaul GAUDILLIÈRE, «The pathological and the artificial: Rückwirkung and the industrial origines of feedback ", in M. D. Laubichler, P. Hammerstein et H.-J. Rheinberger (dir.), Regulation: Current and historical themes in theoretical biology, Cambdridge, MIT Press, sous presse. 
Ce couplage entre industrialisation, pureté et biologisation tendait à marginaliser les savoirs chimiques au sein de la recherche de Schering. Or, paradoxalement, les années de croissance rapide de la production d'hormones sexuelles sont aussi celles de leur caractérisation comme stérö̈des. Pour comprendre cette dissociation entre biochimie et innovation interne, il faut s'intéresser aux échanges scientifiques, matériels et financiers liant le Hauptlaboratorium à une série de partenaires académiques et de compétiteurs industriels. Dans le cas des hormones sexuelles, les plus importants ont été les firmes Ciba et Organon, les services de gynécologie de quelques grands hôpitaux, tels ceux de La Charité à Berlin et de l'hôpital de Koenigsberg, et surtout l'institut de biochimie de la Kaiser Wilhlem Gesellschaft (KWIB) à Berlin-Dalhem. La collaboration avec ce dernier est particulièrement importante pour l'histoire technoscientifique locale puisque le KWIB et son directeur, Adolf Butenandt, ont été des partenaires de longue durée de la firme berlinoise ${ }^{46}$. Ces liens étroits entre un laboratoire universitaire et une entreprise industrielle étaient loin d'être exceptionnels dans le paysage scientifique allemand, non seulement en physique ou chimie mais aussi dans les sciences de la vie ${ }^{47}$.

Cette association a été initiée immédiatement après que Butenandt ait terminé sa thèse sur l'isolement de l'«hormone sexuelle femelle » en 1933. La firme Schering était très intéressée par le protocole de purification mis au point par le jeune biochimiste et négocia la possibilité de le breveter ${ }^{48}$. Avant de venir occuper la direction du KWIB en 1936, Butenandt avait étendu ses travaux à l'ensemble des hormones sexuelles mâles et femelles, associant préparation, analyse de composition chimique, exploration des effets physiologiques chez l'animal. Le premier apport de cette biochimie académique au stock de connaissances exploitées par la firme portait paradoxalement moins sur le versant biologique que sur le versant chimique avec la description des structures (l'assimilation de ces hormones naturelles aux stéroïdes) et l'optimisation des techniques de purification. Mais le système d'échanges progressivement établi entre le laboratoire Butenandt et Schering était multiforme, non limité à la circulation des énoncés d'une part, des financements de l'autre ${ }^{49}$. Les services mutuels avaient ainsi une forte dimension matérielle et technique. Le KWIB fut régulièrement utilisé comme site de test où les physiologistes de la firme pouvaient faire examiner telle ou telle de leur préparation aux effets biologiques inattendus. De même, l'institut de Dalhem bénéficia très largement de l'infrastructure de quantification biologique de Schering (les tests de féminisation et de masculinisation étaient pour la plupart réalisés par le Hauptlaboratorium)

46 - «Adolf BUTENANDT », New dictionary of scientific biographies, New York, Gale, 2008. 47 - Bernd Gausemeier, Natürliche Ordnungen und politische Allianzen. Biologische und biochemische Forschung an Kaiser-Wilhelm-Instituten, 1933-1945, Göttingen, Wallstein, 2005. 48 - Peter KARLSON, Adolf Butenandt. Biochemiker, Hormonforscher, Wissenchaftpolitiker, Stuttgart, Wissenschaftliche Verlaggesellschaft, 1990; Archiv der Max-Planck-Gesellschaft, Butenandt Nachlass: Vertrag zwischen Herrn Prof. Dr A Butenandt und der ScheringKahlbaum Aktiengesellschaft, 17 novembre 1933.

49 - Sur ce système voir Jean-Paul GaudiLLière, «Better prepared than synthesized: Adolf Butenandt, Schering Ag, and the transformation of sex steroids into drugs (1930- 
ainsi que des apports d'hormones ou d'extraits bruts susceptibles de devenir le point de départ de nouveaux projets biochimiques ${ }^{50}$.

Le travail sur la synthèse biologique des stéroïdes sexuels est sans doute le résultat le plus original d'un réseau qui rendait les frontières entre académie, production et commerce très poreuses sans pour autant qu'intervienne, comme chez Madaus, une unité des lieux et des acteurs. Pour les biochimistes des années 1930, l'élucidation des transformations chimiques par lesquelles les cellules de l'organisme sont capables soit de dégrader, soit de synthétiser telle ou telle catégorie de molécules biologiques était un problème essentiel. Dans le cas des stéroïdes, cette biosynthèse semblait aussi complexe que leur structure carbonée. Une part importante des explorations du KWIB a ainsi consisté à rechercher - par synthèse artificielle de molécules ressemblant aux ostrogènes, à la progestérone et à la testostérone - quels pouvaient être les intermédiaires aboutissant, dans les ovaires et dans les testicules, à l'une ou l'autre de ces trois catégories d'hormones. La pratique de purification et d'analyse des hormones biologiques était commune à Schering et au KWIB. Elle se doublait, au sein de l'institut de biochimie, d'un travail systématique de synthèse par modification des radicaux portés par le noyau stéroïde. D'un côté, on y identifiait de nouvelles substances à partir des matériaux de la biologie industrielle, de l'autre, on transformait ces mêmes molécules en analogues et dérivés. Contrairement au criblage qui faisait de la synthèse un mode quasi infini de diversification des candidats à l'action thérapeutique, la chimie organique du KWIB visait, en multipliant ces analogues, à imiter les voies naturelles de biosynthèse pour ensuite, éventuellement, transférer intermédiaires et voies métaboliques ainsi identifiées dans l'enceinte de l'entreprise où ils serviraient à remodeler, sur une base mimétique, les protocoles de fabrication.

Sans équivalent dans la culture de la préparation, cette diversification moléculaire est à l'origine de nombreuses remises en cause des frontières entre l'artificiel et le naturel, le normal et le pathologique, le féminin et le masculin. Donner une place aux très nombreux intermédiaires possibles conduisant de substances carbonées relativement simples aux stéroïdes impliquait, pour Butenandt et ses collègues, de prendre en compte trois types de données : les structures chimiques, les parentés rendues manifestes par les réactions de synthèse du laboratoire et les résultats de tests fonctionnels. Dans les essais standards, les molécules candidates pouvaient ainsi présenter des propriétés femelles ou mâles, voire - pour les stérö̈des trop «éloignés » des hormones naturelles - ni l'une, ni l'autre. La surprise et le problème vinrent de ce qu'une partie des intermédiaires - ceux que Butenandt appellera hormones hermaphrodites - présentait les deux types d'effets à la fois. Si ces ambiguïtés avaient été isolées, on aurait pu les tenir pour une aberration du laboratoire mais le fait que, dans certaines circonstances, mêmes les stéroïdes naturels pouvaient avoir des effets inverses de ceux observés dans les tests de référence,

1946) », Studies in History and Philosophy of the Biological and Biomedical Sciences, 36, 2005, p. 612-644.

50 - Schering se trouva ainsi fournir Butenandt en extraits de glandes de vers à soie utilisées par les chercheurs du KWIB dans une triple quête des voies de synthèse des 
fit qu'il était difficile d'en rester à un simple décalque moléculaire de l'opposition binaire entre les deux sexes. La solution trouvée par Butenandt pour assouplir les frontières du sexe moléculaire sans remettre en cause la notion de déterminisme physio-anatomique du masculin et du féminin consista à faire des transformations métaboliques des étapes dans la voie d'une différenciation hormonale progressive des molécules, des étapes de potentialisation et de spécification sexuelle des stéroïdes. Dans cette perspective, les hormones hermaphrodites pouvaient être considérées comme "précurseur commun» à placer relativement en amont dans une généalogie de molécules qui - arguait Butenandt à la fin des années 1930 - conduisait du cholestérol aux trois types de stéroïdes sexuels ${ }^{51}$.

Ces intermédiaires de synthèse ainsi que les réactions à l'origine de leur généalogie étaient potentiellement des entités industrielles. D’une part, parce ce que dans quelques cas, les analogues d'œstrogènes, de progestérone ou de testostérone présentaient des propriétés susceptibles d'en faire des produits médicalement et commercialement intéressants. Même si une catégorie de dérivés - ceux incluant une chaîne ethinyl - fut effectivement mise sur le marché, cette voie classique de l'innovation pharmaceutique ne fut pas la plus importante ${ }^{52}$. Le travail commun avec Butenandt a essentiellement porté sur le lien entre séquences métaboliques et protocoles industriels, entre synthèse normale des stéroïdes dans le cytoplasme cellulaire et synthèse standard des stéroïdes dans l'usine de Adlershof. À partir de la fin des années 1930 et pendant toute la guerre, Schering réorganisa progressivement son dispositif de production pour faire du cholestérol le point de départ des chaînes de production des Progynon, Proluton et Testoviron. Dans cette perspective, un nombre considérable d'investigations fut consacré à l'un des intermédiaires identifiés par le laboratoire de Butenandt en 1935 - la dehydroandrostérone - qui devint la plate-forme moléculaire commune à l'élaboration des trois groupes de stéroïdes sexuels ${ }^{53}$. En retour, quelques analogues produits par les ingénieurs de Schering finirent dans les schémas d'interconversion entre stéroïdes utilisés au KWIB.

Une dernière remarque sur ces pratiques de la pureté et de la synthèse partielle, les circulations entre Schering et le KWIB et la construction du marché des hormones porte sur la dimension propriétaire des protocoles. Comme on l'a rappelé plus haut, dans l'entre-deux-guerres, les agents thérapeutiques étaient, en Allemagne, exclus de la brevetabilité. Plutôt que de tenter d'obtenir une réforme de cette

pigments occulaires, des hormones d'attraction et des facteurs contrôlant la métamorphose chez les insectes.

51 - Adolf ButenandT, «Ergebnisse und Probleme in der biochemischen Erforschung der Keimdrüsenhormone », Naturwissenschaften, 24, 1936, p. 529-536.

52 - Dans les années 1960, ceux-ci prirent d'autant plus d'importance commerciale qu'ils entraient dans la composition des diverses formules de pilules contraceptives proposées par Schering. Sur cette trajectoire, voir Wolfram FrobEnIUS, Ein Siegezug mit Hindernissen, Berlin, Schering AG, 1989.

53 - Jean-Paul GaudiLlik̀ Schering im Nationalzosialismus ", in W. SchiEdER et A. TRUNK (dir.), Adolf Butenandt und die Kaiser-Wilhelm-Gesellschaft. Wissenschaft, Industrie und Politik im Dritten Reich, Göttingen, Wallstein, 2004, p. 198-245. 
législation, les grandes firmes de la chimie et de la pharmacie la contournèrent en recourant de plus en plus à des brevets protégeant non pas les produits ou les molécules mais les procédés de transformation, les réactions chimiques et leur combinaison ${ }^{54}$. Un attrait des séquences du KWIB tenait précisément à cette possibilité de traduction en demande de brevets ${ }^{55}$. Mais la relation inverse a aussi joué: pour les biochimistes, l'intérêt pour l'élucidation des séquences métaboliques était d'autant plus grand qu'elles étaient utiles et négociables. Au-delà du cas du KWIB, on peut sans grand risque estimer que l'essor remarquable de la biochimie métabolique allemande dans les années 1930 doit beaucoup à la nature biotechnologique de ces connaissances et à leur usage comme actif de propriété intellectuelle.

Analysant la trajectoire de l'insuline, l'historienne de la médecine Christine Sinding a mis en exergue la place que le travail clinique et les formes particulières de standardisation mises en œuvre par les médecins (dans le cas de la correspondance entre diabétologues et la firme Eli Lilly) ont joué dans la normalisation conjointe de l'hormone pancréatique et des thérapies du diabète ${ }^{56}$. Le développement des hormones sexuelles présente des phénomènes analogues avec toutefois cette particularité que le «marketing scientifique » pratiqué par Schering a autant pesé dans la régulation que l'expérience thérapeutique.

Nombre de produits pharmaceutiques de l'entre-deux-guerres étaient associés à une gamme d'indications d'autant plus large que leur cible était définie par une combinaison de symptômes et de maladies. Le phénomène était encore plus net pour les spécialités «physiologiques» tenues pour non toxiques (sauf à des doses très élevées) et agissant sur les fonctions naturelles de l'organisme. En 1934, résumant les usages médicaux des œstrogènes, les deux chefs de laboratoire de Schering dressaient ainsi une liste incluant le traitement des désordres de la «fonction sexuelle » (c'est-à-dire l'ensemble des anomalies du cycle sexuel, les problèmes de stérilité et de gestation) mais également des pathologies «poly-hormonales» (incluant les stéroïdes sexuels et d'autres hormones comme celles de la thyroïde) ainsi que des indications générales comme les troubles de la ménopause, les maladies de peau, les rhumatismes, l'athérosclérose, l'insomnie, les états dépressifs et les psychoses ${ }^{57}$. Toutes ces indications avaient fait l'objet de publications, soit dans les revues médicales, soit dans la revue scientifique de la compagnie, Medisinische Mitteilungen. Mais elles étaient très inégalement stabilisées et standardisées. Celles qui avaient fait l'objet des investissements les plus importants étaient les indications «sexuelles »

\footnotetext{
54 -Arndt Fleischer, Patentgesetzgebung und chemisch-pharmazeutische Industrie im deutschen Kaiserreich (1871-1918), Stuttgart, Deutsche Apotheker Verlag, 1984 ; JeanPaul Gaudillière, "Professional or industrial order? Patents, biological drugs, and pharmaceutical capitalism in early twentieth century Germany ", History and Technology, 24-2, 2008, p. 107-133.

55 - Entre 1925 et 1945, Schering obtint 70 brevets concernant la synthèse des stéroïdes. Comme en témoigne la correspondance avec Butenandt, plus du tiers avait un lien avec les produits et réactions étudiés au KWIB.

56 - Christine SINDING, « Making the unit of insulin: Standards, clinical work and industry, 1920-1925 ", Bulletin of the History of Medicine, 76-2, 2002, p. 231-270.

57 - Karl JunkmanN et Walter Hohlweg, « Über unsere Hormonpräparate », Medizinische Mitteilungen, 6, 1934, p. 130-138.
} 
des oestrogènes et de la progestérone, explorées par une demi-douzaine de cliniciens collaborant régulièrement avec la compagnie.

L'exemple de la clinique gynécologique (Frauenklinik) de l'hôpital universitaire de La Charité à Berlin, dirigée par Carl Kaufmann, illustre la façon dont la régulation industrielle a formaté la clinique pour simultanément définir normalité physiologique et indications. Contrairement aux relations contractuelles et financières avec les biochimistes, les liens avec les cliniciens consistaient en un échange régulier de molécules contre des comptes rendus (le plus souvent oraux) d'observation; les cliniciens restant libres de sélectionner les patients intéressants et de définir les modalités thérapeutiques. Kaufmann s'engagea ainsi dans une exploration de près de dix ans du rôle des hormones dans le cycle sexuel ainsi que de dysfonctionnements comme l'absence ou le caractère irrégulier des règles. Les résultats furent de deux ordres : un cycle normal et des protocoles de traitement de référence. Les expérimentations de Kaufmann sur des patientes souffrant de dysménorrhées montrent ainsi comment les essais d'administration d'abord des œstrogènes puis de combinaison d'œstrogènes et de progestérone ont débouché sur un «régime» mensuel standard d'administration de ces composés, destiné à rétablir les variations physiologiques des deux types d'hormones. Contrairement à l'idée selon laquelle le normal explique le pathologique, le tableau des changements de concentration hormonale caractéristiques de ce qui devint le cycle sexuel de référence n'a pas été la condition de possibilité de ce régime mais le résultat parallèle d'un seul et même processus de normalisation par la pratique clinique ${ }^{58}$. Mis au point dans le cadre des échanges avec Kaufmann, publié à plusieurs occasions dans la presse médicale dans des articles repris par Medizinische Mitteilungen, le régime œstrogène-progestérone a ensuite été intégré à l'ensemble du dispositif de marketing scientifique, c'est-à-dire aux brochures, notices et quasi-manuels distribués par Schering auprès des gynécologues.

\section{La préparation entre ordre professionnel et ordre industriel}

La comparaison entre les activités de recherche et de production des deux firmes Madaus et Schering, et plus spécifiquement entre leur mode d'industrialisation des extraits de plantes et des extraits de glandes endocrines, a été utilisée dans cet article comme un moyen d'approcher les deux grandes formes de régulation qui ont dominé l'histoire de la pharmacie au début du $\mathrm{XX}^{\mathrm{e}}$ siècle, en focalisant la discussion sur les trajectoires de deux types de préparations pharmaceutiques transformées en spécialités médico-commerciales dans un même contexte intellectuel, social et politique.

58 - Sur Kaufmann, voir M. SCHLUENDER, «Reproduktionen, Experimentalisierung der Geburtshilfe zwischen 1900 und 1930 », op. cit.; sur la standardisation clinique, Jean-Paul Gaudillitere, "Genesis and development of a biomedical object: Styles of thought, styles of work and the history of the sex steroids ", Studies in the History and Philosophy of the Biological and Biomedical Sciences, 35-3, 2004, p. 525-543. 
Le premier intérêt de cette comparaison est en effet le constat qu'en dépit des histoires contrastées de ces firmes et des différences de nature entre ces préparations, leurs trajectoires présentent de grandes similitudes. Dans les deux entreprises, il s'agissait de fabriquer des médicaments biologiques avec pour horizon commun la culture pharmaceutique de la préparation. Celle-ci constituait un point de référence tant du point de vue de la production des connaissances (sur les substances, leur origine et leur combinaison, les effets physiologiques et toxiques) qu'en termes de fabrication (les protocoles de la pharmacopée, les formulations). Dans les deux cas, la mise au point des spécialités a largement fait appel à cette culture privilégiant non pas la mobilisation des savoir-faire de la chimie organique (ce qu'une certaine histoire de la pharmacie modelée sur le cas de IG Farben laisserait penser) mais ceux de la physiologie - animale et végétale - ainsi que de la mécanique.

Dans les deux cas aussi, l'industrialisation des préparations correspond à un changement de nature des produits, des marchés et des acteurs qui y opèrent. Celui-ci renvoie à la montée de l'entreprise pharmaceutique capitaliste comme alternative aux petites unités de production issues de l'officine, mais aussi à l'émergence d'un nouveau mode de régulation de la fabrication, de la vente et des usages des médicaments (voir tableau). Par rapport à la régulation professionnelle, la régulation industrielle privilégie des pratiques de standardisation faisant appel à la multiplication des essais biologiques, au contrôle systématique des matières premières et des produits, à la formalisation des protocoles de fabrication dont on surveille ensuite l'observance. Un élément essentiel mis en exergue par les activités de Madaus et de Schering est que cette mise en équation de la spécialité avec le développement de pratiques d'homogénéisation, de production de masse, de qualification de la valeur clinique et de contrôle de la composition ne passait pas nécessairement par le recours aux dispositifs de purification et d'isolement des substances. Les activités de Madaus et Schering indiquent aussi en quoi cette régulation industrielle n'est pas qu'une quête de la productivité et du profit par l'organisation scientifique des manipulations mais correspond à un mode particulier de construction des marchés et introduit de nouvelles modalités de définition des pratiques médicales légitimes. Deux éléments sont ici importants : d'une part le fait que les protocoles de production, éventuellement copiés des réactions biochimiques intracellulaires, ont été systématiquement transformés en actifs de propriété intellectuelle sans que l'on change les lois sur l'absence de brevet de médicaments; d'autre part le fait que les échanges entre firmes et médecins sont devenus plus intenses, que la standardisation clinique est venue relayer la standardisation des produits, que des modes de marketing faisant très largement appel aux informations scientifiques produites, collectées et mises en forme par les firmes sont venues concurrencer le recours aux annonces publicitaires.

Une fois ceci posé, les différences entre les deux firmes et les trajectoires de leurs produits sont tout aussi intéressantes à considérer. Les activités de Madaus révèlent en effet une articulation entre régulation industrielle et régulation professionnelle beaucoup plus poussée que cela n'était le cas chez Schering. Cela tient d'une part à l'insertion de Madaus dans les réseaux de la médecine biologique «populaire» et par voie de conséquence à son intérêt pour une grande diversité 
de préparations et de thérapies «traditionnelles » utilisées localement par médecins diplômés et thérapeutes. Mais cette intégration est aussi la conséquence de la priorité accordée par G. Madaus et ses employés scientifiques aux mélanges, à l'étude des synergies entre substances, à la mobilisation des savoirs botaniques et à l'exploration des maladies comme phénomène physiologique global. Nombre de pratiques de la firme sont ainsi directement issues du laboratoire de "matière médicale ", par exemple le recours aux pharmacopées comme source de recettes et modèle d'organisation des connaissances. De même, chez Madaus, la modélisation animale a tout autant servi à standardiser qu'à modifier les formulations sans pour autant déboucher sur une exploration des fonctions physiologiques « fondamentales » des organismes animaux ou végétaux.

À l'inverse, Schering a inventé un dispositif d'innovation biologique centré sur l'isolement, la purification et la synthèse partielle d'entités biologiques définies au niveau moléculaire. Cette priorité accordée au niveau moléculaire est commune avec le modèle de recherche industrielle adopté chez IG Farben et basée sur l'association entre synthèse organique de milliers de molécules et criblage pharmacologique. La stratégie de Schering s'en distingue radicalement par la mobilisation des sciences du vivant. L'histoire de la compagnie, son succès initial dans une industrialisation de la pharmacie visant à la fabrication des produits « intermédiaires » utilisés par les pharmaciens d'officine a certainement une part dans ce choix. La molécularisation des hormones a toutefois suivi une dynamique propre, liée à la mise en place d'un dispositif de recherche liant la pharmaco-physiologie «maison » aux investigations biochimiques et cliniques menées par des partenaires académiques. Un des corrélats de cette trajectoire de molécularisation est d'avoir renforcé le poids de la régulation industrielle. Ainsi, chez Schering, à la différence de ce qui se passait chez Madaus, brevets de procédés, essais biologiques, standardisation clinique sont devenus les instruments courants d'un même processus de construction du marché et de définition des usages médicaux.

Pour conclure cette exploration, rappelons que les formes professionnelle et industrielle de la régulation du médicament correspondent à des agencements entre valeurs, acteurs, types de savoirs et outils d'intervention. L'intérêt d'une classification de ces arrangements et de leur succession est en premier lieu heuristique. Il s'agit de se doter d'outils pour analyser des configurations locales de pratiques qui font intervenir des actions relevant de l'une ou l'autre de ces formes de régulation mais qui sont aussi à l'origine de leur différenciation et de leur hiérarchisation. En ce sens, il n'y a pas d'ontologie de la régulation mais un travail local, spécifique, de mise en œuvre, de combinaison et de séparation des dispositifs. Même s'il faut rester prudent dans nos tentatives de généralisation, cet exercice typologique permet d'interroger à un niveau plus global l'histoire du médicament, les rapports qu'elle entretient avec les changements, au cours du XX⿳亠丷厂 siècle, des relations entre sciences, médecine et société. 